\title{
Drugs that may injure the respiratory system
}

\author{
P. Foucher*, M. Biour**, J.P. Blayac ${ }^{+}$, P. Godard + , C Sgro+e, \\ M. Kuhn $¥$, J.M. Vergnon $\neq \neq$, D. Vervloet\#, P Pfitzenmeyer\#\#, \\ M. Ollagnier\$, Ch. Mayaud**, Ph. Camus*
}

The above authors have founded a clinical study group known as the GEPPI (Groupe d'Etudes de la Pathologie Pulmonaire Iatrogene. Address: Professor Ph. Camus, Service de Pneumologie, CHU, BP 1542, F-21034 Dijon, France; fax: 333802932 51; e-mail: pneumo.dijon@ planetb.fr). The purpose of this group is to provide information regarding individual cases, collect and update literature on drug-induced lung disease, publish updated lists of offending compounds, and formulate warnings when new side-effects of drugs are recognized. Thus, the present table may be subjected to update in the future in this Journal.

The number of drugs which have been shown to damage the respiratory system in some instances continues to increase. The clinical pattern of involvement from drugs is diverse and difficult to memorize accurately. Table 1 is designed to provide the information currently available on such drugs in a readily accessible form.

A good way of obtaining broad information on druginduced respiratory disease is to refer to general articles and reviews on the topic. Many such papers have been available in the literature in the recent years [1-37].

Another method is to scrutinize the literature regarding any given drug. We considered that the preparation of a table listing most drugs capable of injuring the respiratory system was appropriate. The present table includes: 1) generic drug name (no trade name is provided); 2) clinical pattern of involvement, according to the types described in the legend; 3 ) a rough estimate of frequency of the adverse effect from each compound $(*$ : isolated case reports which await confirmation; **: about 10 cases available; $* * *$ : $20-100$ cases available; ****: $>100$ cases reported); 4) relevant references. Obviously, not all references for a given compound have been mentioned, because the overall list would have been too long. For example, references regarding pulmonary adverse effects from bleomycin total 114, and 135 for nitrofurantoin.
No attempt has been made to validate the case histories quoted here.

Often, most if not all drugs from the same pharmacological category are likely to induce the same adverse effect in the lung. As an example, angiotensin-converting enzyme (ACE) inhibitors may induce cough [38], $\beta$-blockers may induce bronchospasm [35], nonsteroidal anti-inflammatory drugs may induce bronchospasm or eosinophilic pneumonia [39], and ergoline drugs may induce chronic pleural thickening or effusion [40].

A few compounds have been listed despite the fact that they were recalled or withdrawn from the market earlier (e.g. aminorex, hexamethonium, mecamylamine, practolol or tryptophan). There are several reasons for this: 1) disease from drugs such as I-tryptophan leave long-term sequelae and patients may still be seen with persistent illness from the drug; 2) the clinical picture (e.g. bronchiolitis obliterans organizing pneumonia (BOOP)) from classic drugs, such as hexamethonium or meca-mylamine, may be seen with newer drugs, such as penicillamine, gold or amiodarone; and 3) clinical pictures long thought to have vanished (e.g. pulmonary hypertension from the appetite suppresant, aminorex) have regained interest because similar newer compounds (e.g. fenfluramine) may induce the same adverse effect [41].

Contraceptive pills may induce varied changes in the pulmonary circulation [42-44], but the aetiological link is too weak to make it possible to blame one particular formulation or brand.

The field of drug-induced pulmonary infections and cancer, as well as the complications of illicit drug use and abuse have been deliberately omitted.

This list of drugs is available on a continuously updated Web page at: http://www.pneumotox.com/lungdrug.

For further comments, see the editorial in this issue of the Journal.

The summary of available information on drugs that may injure the respiratory system appears on the following pages.

Services de Pneumologie *Dijon, Montpellier, ${ }^{+}$St Etienne, \#Marseilles, France. Services de Pharmacovigilance **Paris, ${ }^{+}$Montpellier, ${ }^{++}$Dijon, ¥Chur (Switzerland), St Etienne\$. \#\#Service de Gériatrie, Dijon, France 
Table 1. - Summary of information available concerning drugs that may injure the respiratory system

\begin{tabular}{|c|c|c|c|}
\hline Drug & Clinical pattern & Frequency & [Ref.] \\
\hline acebutolol & I b, d; V a, b, c, d & $* *$ & [45] \\
\hline ACE inhibitors & I c; IV a, d; VIII a & $* * * *$ & {$[46-50]$} \\
\hline acetaminophen & I c; IV a & * & {$[51-53]$} \\
\hline acetylcysteine & IV a & * & [54] \\
\hline acetylsalicylic acid (aspirin) & I c; II a, b; III a; IV a, b; VII b; X & $* * *$ & {$[55]$} \\
\hline acrylate & VI a & $* *$ & [56] \\
\hline acyclovir & $\mathrm{I} \mathrm{b} ; \mathrm{Va}, \mathrm{b}, \mathrm{c}$ & $*$ & {$[57]$} \\
\hline adenosine & IV a & $*$ & {$[58]$} \\
\hline adrenaline (epinephrine) & II a & * & [59] \\
\hline albuterol (see salbutamol) & II a & * & {$[60-62]$} \\
\hline allopurinol & VI d & $*$ & [63] \\
\hline aminoglycoside antibiotics & IX a & $* *$ & {$[64]$} \\
\hline aminorex (recalled in early 1970) & VI b & $* * * *$ & {$[65]$} \\
\hline amiodarone & I b, c, d, g, k; IV a; V a, c, d & $* * * *$ & {$[21,22,66-74]$} \\
\hline amitryptyline & II a, b & * & {$[75]$} \\
\hline amphotericin B & $\mathrm{I} d ; \mathrm{II} \mathrm{a}, \mathrm{b}$ & * & [76-79] \\
\hline ampicillin & I b, c & * & {$[80]$} \\
\hline amrinone & I b & * & [81] \\
\hline antilymphocyte globulin & II a & * & [82] \\
\hline $\mathrm{L}$-asparaginase & IV a, b; VI a & $*$ & {$[83-85]$} \\
\hline aurothiopropanosulphonate (gold salt) & I a, b, c, d; IV c & $* * *$ & {$[86-93]$} \\
\hline azapropazone & $\mathrm{I} \mathrm{b}$ & * & {$[39,94]$} \\
\hline azathioprine & $\mathrm{I} b$ & $* *$ & {$[95]$} \\
\hline BCG therapy & $\mathrm{I} b$ & $* *$ & [96] \\
\hline bepridil & I g & $*$ & [97] \\
\hline betahistine & IV a & $*$ & [98] \\
\hline bleomycin & b, c, d, g, k; II b; V f; VI c; VII a; XI b & $* * * *$ & [99-107] \\
\hline blood transfusions & II a & $* * *$ & [108-114] \\
\hline bromocriptine & $\mathrm{V}$ a, c & $* * *$ & {$[40]$} \\
\hline bucillamine & I c; IV d & $*$ & {$[115]$} \\
\hline busulphan & I e, g; VI c & $* * *$ & [20] \\
\hline cabergoline & $\mathrm{V} \mathrm{a}, \mathrm{c}$ & $*$ & [40] \\
\hline calcium salts & I i & $* *$ & {$[116,117]$} \\
\hline captopril & I b, c, f; IV d; VIII a & $* * * *$ & {$[50]$} \\
\hline carbamazepine & I b, c, k; II a; III a; V d; VII a, b; X & $* * *$ & {$[46,118-125]$} \\
\hline carmustine & I g; II b; V a, f; VI c & $* * *$ & {$[32,126]$} \\
\hline celiprolol & I a & $*$ & {$[45]$} \\
\hline cephalosporins & I c, d; IV a, b & $*$ & [127-129] \\
\hline chlorambucil & $\mathrm{I} \mathrm{b}$ & $* *$ & [130] \\
\hline chlorpromazine & I c; II a; V d; VI a & $*$ & {$[131,132]$} \\
\hline chlorpropamide & I c & $*$ & {$[133]$} \\
\hline ciprofloxacin & IV b & $*$ & [134] \\
\hline clofazimine & $\mathrm{I} \mathrm{h}$ & $*$ & {$[135]$} \\
\hline clofibrate & I c; V d & $*$ & [136] \\
\hline clomiphene & II a, b; V a; VI a & $* *$ & {$[137,138]$} \\
\hline clonidine & $\mathrm{Vd}$ & $*$ & [139] \\
\hline colchicine & II a; X & $*$ & {$[140]$} \\
\hline contraceptives (oral) & VI b, c & $* * *$ & {$[43,44]$} \\
\hline contrast media & I c; II a, b; IV a, b & $* * * *$ & [141-145] \\
\hline co-trimoxazole & I b, c; II a & $* *$ & {$[146,147]$} \\
\hline cromoglycate & I b, c & $*$ & {$[148]$} \\
\hline curare & IV a, b & $* * *$ & {$[129,149]$} \\
\hline cyclophosphamide & I c, g; II a; IV a, b. V a & $* * *$ & {$[150-154]$} \\
\hline cyclosporin & I j; II a; III a; VI b & $*$ & [155-159] \\
\hline cytarabine & II b; III a & $* *$ & [160-164] \\
\hline dantrolene & $\mathrm{V} \mathrm{b}$ & $*$ & {$[165,166]$} \\
\hline dapsone & XI a & $*$ & [167] \\
\hline deferoxamine & I c; II a, b; VI a & $* *$ & [168-171] \\
\hline desipramine & I c; IV a & $* *$ & {$[172-174]$} \\
\hline dexamethasone & II a & $* *$ & {$[175]$} \\
\hline dexfenfluramine & $\mathrm{I} b ; \mathrm{VI} b$ & $* * *$ & {$[176-178]$} \\
\hline diclofenac & I c, IV a & $* *$ & {$[39,179,180]$} \\
\hline
\end{tabular}




\begin{tabular}{|c|c|c|c|}
\hline Drug & Clinical pattern & Frequency & [Ref.] \\
\hline dihydralazine & $\mathrm{I} b, \mathrm{~d} ; \mathrm{V} \mathrm{a}, \mathrm{d}$ & ** & {$[39,181-185]$} \\
\hline dihydro-5-azacytidine & XI c & $* *$ & [186] \\
\hline dihydroergocristine & $\mathrm{V} \mathrm{a}, \mathrm{c}$ & $*$ & {$[40]$} \\
\hline dihydroergocryptine & $\mathrm{I} b, \mathrm{~d}$ & * & [40] \\
\hline dihydroergotamine & I d & * & [40] \\
\hline diltiazem & II a & $*$ & {$[187]$} \\
\hline I-DOPA & VII b; IX b & * & {$[188,189]$} \\
\hline dothiepin & $\mathrm{I} b, \mathrm{~g}$ & $*$ & {$[190,191]$} \\
\hline enalapril & IV d; VIII a & $* *$ & {$[192,193]$} \\
\hline ergometrine & IV a & $*$ & [194] \\
\hline ergotamine & $\mathrm{I} \mathrm{b} ; \mathrm{V}$ a & $* *$ & {$[40]$} \\
\hline erythromycin & II $\mathrm{a}, \mathrm{b}$ & * & [195] \\
\hline ethambutol & I c & * & [196] \\
\hline ethchlorvynol & II a; $\mathrm{V}$ a & $*$ & {$[131,197]$} \\
\hline etoposide & XI b (due to coronary spasm) & $* * *$ & {$[198,200]$} \\
\hline fenbufen & I c, VII a & $*$ & {$[39,200-202]$} \\
\hline fenfluramine & I b; VI b & $* * *$ & {$[176-178]$} \\
\hline fenoprofen & I c & $*$ & {$[39,205]$} \\
\hline flecainide & $\mathrm{I} b$ & $*$ & [204] \\
\hline floxuridine & $\mathrm{I} b$ & $* *$ & [205] \\
\hline fludarabine & I a; II b & $*$ & {$[206,207]$} \\
\hline fluoxetine & $\mathrm{I} \mathrm{b}$ & $* *$ & [209-212] \\
\hline flurbiprofen & IV a & $*$ & [208] \\
\hline furazolidone & I c & $*$ & [213] \\
\hline glafenine & $1 \mathrm{c}$ & $*$ & [214] \\
\hline glibenclamide & III a; X & * & [215] \\
\hline $\mathrm{G}(\mathrm{M})-\mathrm{CSF}$ & I b, c; II b & $* * *$ & [216-211] \\
\hline haloperidol & II b & $*$ & [222] \\
\hline heparin & II $b$ & $*$ & [223] \\
\hline hexamethonium (discontinued) & I d & $* * *$ & [224] \\
\hline hyralazine & I b, d; III a; V a, d; VI b & $* *$ & [181-183, 225-227] \\
\hline hydrochlorothiazide & I b; II a, b & $* * *$ & [228-234] \\
\hline hydrocortisone & IV b & $*$ & [129] \\
\hline hydroxyquinoline & $\mathrm{I} \mathrm{b}$ & $*$ & {$[235]$} \\
\hline hydroxyurea & I b & $* *$ & {$[236]$} \\
\hline ibuprofen & I c, II a & $*$ & {$[39,202,237,238]$} \\
\hline ifosfamide & $\mathrm{I} \mathrm{b}$ & * & [239] \\
\hline imipramine & I c & $* *$ & {$[240]$} \\
\hline immunoglobulins (IVIG) & II a; IV b & $* *$ & [129-241] \\
\hline indomethacin & IV b & $*$ & [129] \\
\hline interferon alpha & $\mathrm{I} \mathrm{b}, \mathrm{d}, \mathrm{e}$ & $* *$ & [242-245] \\
\hline interleukin 2 & II a, b; IV a, b; IV a, b & $* *$ & [246-248] \\
\hline iodine, radiographic contrast media & I c, j; II a; V a; IV b & $* * * *$ & [141-145] \\
\hline isoniazid & $\mathrm{I} \mathrm{c} ; \mathrm{V} \mathrm{d}$ & $* *$ & {$[249,252]$} \\
\hline isotretinoin & I c, IV a; V b & $* *$ & {$[253,254]$} \\
\hline itraconazole & $\mathrm{V} \mathrm{c}$ & & [255] \\
\hline labetalol & I g & $*$ & {$[256,257]$} \\
\hline leuprorelin & II b & $*$ & [258] \\
\hline lidocaine & II a; IV b & $*$ & {$[129,259]$} \\
\hline lisinopril & VIII a & $* *$ & {$[260]$} \\
\hline lisuride & $\mathrm{V}$ a, c & $*$ & {$[261]$} \\
\hline lomustine & I g, VIc & $* *$ & {$[32]$} \\
\hline loxoprofen & I c; IV a & $*$ & {$[39,262,263]$} \\
\hline lupron & $\mathrm{V} \mathrm{e}$ & * & [264] \\
\hline maprotiline & $\mathrm{I} b$ & * & [265] \\
\hline mecamylamine (discontinued) & I d & $* *$ & [266] \\
\hline medroxyprogesterone & I g & * & [267] \\
\hline melphalan & I c, g; IV a & $* *$ & {$[268-271]$} \\
\hline mephenesin & I c & $*$ & {$[272]$} \\
\hline mercaptopurine & I b & $*$ & {$[273]$} \\
\hline mesalamine & I c, d; XI b & ** & {$[274]$} \\
\hline metapramine & I b & $*$ & {$[275]$} \\
\hline methadone & II a & * & {$[276,277]$} \\
\hline methotrexate & I a, b, c; II a; IV b; V a; XI b & $* * * *$ & {$[29,278-281]$} \\
\hline
\end{tabular}




\begin{tabular}{|c|c|c|c|}
\hline Drug & Clinical pattern & Frequency & [Ref.] \\
\hline methyldopa & V d; VII b & $* *$ & {$[30,282]$} \\
\hline methysergide & I b; V a, c; VIId, XI b & $* *$ & {$[40,283,284]$} \\
\hline metoclopramide & IV a & $*$ & [285] \\
\hline metoprolol & IV a; VI b & $*$ & [286] \\
\hline metronidazole & I c & * & {$[287]$} \\
\hline miconazole & II $\mathrm{b}$ & $*$ & [288] \\
\hline minocycline & I c, d, k; VII b; X; XI b, c & $* * *$ & [289-295] \\
\hline mitomycin $\mathrm{C}$ & I b, g; II b, c; III a; VI b & $* * *$ & [296-304] \\
\hline mitoxantrone & $\mathrm{I} \mathrm{b}$ & $*$ & [305] \\
\hline moxalactam & III a & $*$ & [306] \\
\hline nadolol & $\mathrm{I} b$ & * & [307] \\
\hline naftidrofuryl & IV a & $*$ & \\
\hline nalbuphine & II a & $* *$ & {$[308,309]$} \\
\hline nalfon & Ic & $*$ & [203] \\
\hline nalidixic acid & I c & $*$ & {$[310]$} \\
\hline naloxone & II a & $* *$ & {$[311,312]$} \\
\hline naproxen & I c, IV a & $*$ & {$[39,180,202,313]$} \\
\hline nicergoline & $\mathrm{V} \mathrm{a}, \mathrm{c} ; \mathrm{XI} \mathrm{b}$ & $* *$ & [40] \\
\hline nilutamide & I a, b, c, d & $* * *$ & {$[35,37]^{+}$} \\
\hline nitrofurantoin & d, e; III a; IV a, b; V a, d; VI d; VIII b; X; XI b, c & $* * * *$ & {$[93,314-321]$} \\
\hline nitroglycerin & II a & * & [322] \\
\hline nitrosoureas & I g, VI c & $* * *$ & {$[32,323]$} \\
\hline nomifensine & $\mathrm{I} b, \mathrm{c}$ & $*$ & {$[324,325]$} \\
\hline NSAIDs & I c; IV a; X & $* * *$ & [39] \\
\hline noramidopyrine & II a & $*$ & [326] \\
\hline oxprenolol & I b, IV a; V a, c & * & {$[45,327,328]$} \\
\hline oxyphenbutazone & $\mathrm{I} \mathrm{b}$ & $*$ & {$[39,329]$} \\
\hline paclitaxel & I b; IV a & $* *$ & {$[330,331]$} \\
\hline para(4)-aminosalicylic acid (PAS) & I c; II a & $* *$ & {$[274,332]$} \\
\hline paraffin (mineral oil) & $I \mathrm{j}$ & $* * * *$ & [333-337] \\
\hline penicillamine & I b, c, d, g; III b; IV a, c; V c & $* * *$ & {$[338,339]$} \\
\hline penicillins & I c; IV a, b; VI d; VII a, b & * & {$[129,340-347]$} \\
\hline pentamidine & I c; IV a & * & {$[348,349]$} \\
\hline perindopril & I c & * & [47] \\
\hline phenylbutazone & I c; II a; VII a & * & {$[39,350-352]$} \\
\hline phenytoin & I b, c, f, k; II a, c, d; VI a, d; VII a, b & $* * *$ & {$[353-360]$} \\
\hline piroxicam & I c & $*$ & [39] \\
\hline pituitary snuff & I b & * & [361] \\
\hline polyethylene glycol & II b; IV a & $*$ & {$[362]$} \\
\hline practolol (recalled) & $\mathrm{I} b ; \mathrm{V} \mathrm{b}, \mathrm{c}$ & $* * *$ & {$[45,363]$} \\
\hline praziquantel & $\mathrm{V}$ a & $*$ & [364] \\
\hline procainamide & I b; V a; d; VI a & $* * *$ & {$[365-368]$} \\
\hline procarbazine & $\mathrm{I} a, b$ & * & [369-373] \\
\hline propafenone & IV a & * & [374] \\
\hline propoxyphene & II a & $*$ & {$[375]$} \\
\hline propranolol & I c; V a, d; VI b & $* *$ & {$[45,286,376,377]$} \\
\hline propylthiouracil & I c, k; II b; V a, b; VI d & $* *$ & [378-381] \\
\hline prostglandin F2- $\alpha$ & IV a & * & [382] \\
\hline protamine & IV b; VI b & $* *$ & {$[129,383]$} \\
\hline pyrimethamine-dapsone & I c; X & $*$ & [384-387] \\
\hline pyrimethamine-sulphadoxine & I b, c; II a & * & {$[385,387]$} \\
\hline quinidine & I b; III a & * & {$[388,389]$} \\
\hline radiations & I b, d, g; II b; V a, c, f; VI c; VII d & $* * * *$ & [390-397] \\
\hline retinoic acid & I b; II a, b & $* *$ & [398-400] \\
\hline ritodrine & II a & $* *$ & {$[60]$} \\
\hline salbutamol & II a; IV a & $* * *$ & {$[60-62]$} \\
\hline simvastin & $\mathrm{I} \mathrm{b} ; \mathrm{d} ; \mathrm{V}$ a & $*$ & [401] \\
\hline steroids & VII c; IX a & $* * *$ & {$[402,403]$} \\
\hline streptokinase & IIb; III a; IV a & $* *$ & [404] \\
\hline streptomycin & I c & ** & {$[249,405]$} \\
\hline sulindac & $I \mathrm{c} ; \mathrm{X}$ & $*$ & {$[39,414-417]$} \\
\hline sulphamides-sulphonamides & I b, c; II a, b; IV a, b; V d; VI d; VII b & $* * *$ & {$[30,406-413]$} \\
\hline
\end{tabular}

+ see also: Pfitzenmeyer P, Foucher P, Piard F, et al. Nilutamide pneumonitis: a report on eight patients. Thorax 1992; 47: 622-627. 


\begin{tabular}{|c|c|c|c|}
\hline Drug & Clinical pattern & Frequency & [Ref.] \\
\hline sulphasalazine & I b, c, e; II a; V b, d; VI d & $* * *$ & {$[274,409-413]$} \\
\hline tamoxifen & I c, g; II a; IV a; VI a & $*$ & {$[303,418]$} \\
\hline terbutaline & II a & $* *$ & [60] \\
\hline tetracycline & I c; V d & $*$ & {$[291,419,420]$} \\
\hline thiopental & IV b & $* *$ & [129] \\
\hline tiopronin & I b; IV c & $*$ & {$[421,422]$} \\
\hline TNF-alpha & II a; III a & $* *$ & {$[423,424]$} \\
\hline tolfenamic acid & I c & $*$ & {$[39,425]$} \\
\hline transfusions (blood, leucocytes) & II a & $* * *$ & {$[108,109,111-114,426]$} \\
\hline triazolam & II a & $*$ & [427] \\
\hline trimethoprim & I c; II a; VII a & $* *$ & {$[146,147]$} \\
\hline trimipramine & $\mathrm{I} \mathrm{c} ; \mathrm{V} \mathrm{b,f}$ & $* *$ & [428] \\
\hline troleandomycin & I c & $*$ & {$[346]$} \\
\hline I-tryptophan (recalled) & I c; VI b, d & $* * * *$ & [429-432] \\
\hline D-tubocurarine & IV a, b & $* * *$ & [129] \\
\hline urokinase & III a; IV a & $*$ & [433] \\
\hline vancomycin & IV b & $*$ & [129] \\
\hline vasopressin & II a & $*$ & {$[434]$} \\
\hline vinblastine & I c, k; II b; IV a, b & $* * *$ & {$[299,435-438]$} \\
\hline vindesine & I b, IV a, b & $* *$ & {$[438,439]$} \\
\hline venorilbine & II a; IV a & $*$ & [440] \\
\hline vitamin D & I i & $*$ & [441] \\
\hline warfarin & II a; V e; VIIIb & $* * *$ & [422-445] \\
\hline zomepirac & IVa & $*$ & [446] \\
\hline
\end{tabular}

ACE: angiotensin-converting enzyme; BCG: bacille Calmette-Guérin; L-DOPA: L-dihydroxyphenylalanine; G/MCSF; granulocyte/macrophage colony-stimulating factor; IVIG: intravenous immunoglobulins; NSAIDS: nonsteroidal anti-inflammatory drugs; TNF- $\alpha$ : tumour necrosis factor- $\alpha$.

I: interstitial lung disease (with diffuse (D) or focal (F) roentgenological changes). a) acute hypersensitivity pneumonitis and respiratory failure (may transiently behave like adult respiratory distress syndrome (ARDS)) (D); b) subacute interstitial lung disease (nonspecific; often lymphocytic bronchoalveolar lavage (BAL) pattern) (D); c) pulmonary infiltrates and eosinophilia (D, F)\$; d) organizing pneumonia \pm bronchiolitis obliterans (BOOP) (D, F); e) desquamative interstitial pneumonia (DIP) (D)\$; f) lymphocytic interstitial pneumonia (LIP) (D) ${ }^{\$}$; g) pulmonary fibrosis (D) $\$$; h) subclinical cytological changes in BAL cell composition; i) pulmonary calcification (D)\$; j) mineral oil pneumonia (F-D)\$; k) multiple lung nodules (usually, focal BOOP or, less often, vasculitis); \$: opacities tend to predominate in basilar regions; \$: opacities tend to predominate in apices.

II: pulmonary oedema. a) acute pulmonary oedema; b) acute permeability oedema often accompanied by ARDS; c) acute permeability oedema, ARDS and the haemolytic and uraemic syndrome (almost specific to mitomycin C).

III: pulmonary haemorrhage. a) alveolar haemorrhage; b) Goodpasture-like syndrome.

IV: airways disease. a) bronchospasm; b) bronchospasm and anaphylactic shock; c) bronchiolitis obliterans; d) cough. $\mathrm{V}$ : pleural changes. a) pleural effusion; b) eosinophilic pleural effusion; c) thickening \pm pericardial thickening/effusion; d) pleural/pericardial thickening or effusion and positive antinuclear/antihistone antibodies, i.e. the drug-induced lupus syndrome; e) haemothorax; f) pneumothorax.

VI: vascular changes. a) thromboembolic disease (with or without antiphospholipid antibodies); b) pulmonary hypertension; c) pulmonary veno-occlusive disease; d) vasculitis.

VII: mediastinal changes. a) enlarged hilar/mediastinal lymph nodes; b) angioimmunoblastic lymphadenopathy-like syndrome/lymphoma; c) mediastinal fatty infiltration (mediastinal lipomatosis); d) sclerosing mediastinitis.

VIII: large airways involvement. a) acute upper airway obstruction and laryngeal oedema (see also bronchospasm and anaphylaxis under IVb); b) upper airway obstruction from peritracheal haemorrhage.

IX: muscle and nerves. a) lowered force generation; b) disordered breathing or respiratory movements (respiratory dyskinesia).

$\mathrm{X}$ : constitutional/systemic symptoms. Systemic hypersensitivity syndrome with a combination of skin rash, lymph node enlargement, eosinophilia, changes in liver chemistry and/or mental disturbances.

XI: varied effects. a) methaemoglobinaemia, cyanosis, elevated blood nitrates and respiratory failure; b) acute chest pain; c) acute chest pain and interstitial pneumonitis.

\section{References}

1. Davies PDB. Drug-induced lung disease. Br J Dis Chest 1969; 63: 57-70.

2. Ansell G. Radiographical manifestations of drug-induced disease. Clin Radiol 1969; 20: 133-148.

3. Rosenow ECI. The spectrum of drug-induced pulmonary disease. Ann Intern Med 1972; 77: 977-991.

4. Whitcomb ME. Drug-induced lung disease. Chest 1973; 63: $418-422$.
5. Uthgenannt H, Lübbers P, Gappmayer K. Allergische Reaktionen der Lunge durch Arzneimittel. Forschr Roentgenstr 1973; 118: 125-136.

6. Lippmann ML. Pulmonary reactions to drugs. Med Clin North Am 1977; 61: 1353-1367.

7. Sostman HD, Matthay RA, Putman CE. Cytotoxic druginduced lung disease. Am J Med 1977; 62: 608-615.

8. Tucker AS, Newman AJ, Alvorado C. Pulmonary, pleural and thoracic changes complicating chemotherapy. Radiology 1977; 125: 805-809. 
9. Rosenow ECI. Drugs that may induce pulmonary disease. Geriatrics 1978; 33: 64-73.

10. Whitcomb ME, Domby WR. Drug-induced lung disease. Primary Care 1978; 5: 411-423.

11. Willson JKV. Pulmonary toxicity of antineoplastic drugs. Cancer Treat Rep 1978; 62: 2003-2008.

12. Leuenberger P. Affections respiratoires d'origine médicamenteuse. Schweiz Rundsch Med Prax 1979; 68: 1732-1739.

13. Weiss RB, Muggia FM. Cytotoxic drug-induced pulmonary disease: update 1980. Am J Med 1980; 68: 259-266.

14. Sostman HD, Putman CE, Gamsu G. Diagnosis of chemotherapy lung. Am J Roentgenol 1981; 136: 33-40.

15. Weiss RB, Poster DS, Penta JS. The nitrosoureas and pulmonary toxicity. Cancer Treat Rev 1981; 8: 111-125.

16. Myers JL. Diagnosis of drug reactions in the lung. Monogr Pathol 1993; 36: 32-53.

17. Muggia FM. Louie AC, Sikic BI. Pulmonary toxicity of antitumour agents. Cancer Treat Rev 1983; 10: 221-243.

18. White JP, Ward MJ. Drug-induced adverse pulmonary reactions. Adv Drug React Acute Pois Rev 1985; 4: 183-211.

19. Rosenow ECI. Drug-induced bronchopulmonary pleural disease. J Allergy Clin Immunol 1987; 80: 780-787.

20. Massin F, Fur A, Reybet-Degat O, Camus P, Jeannin L. La pneumopathie du busulfan. Rev Mal Respir 1987; 4: 3-10.

21. Martin WJ II, Rosenow EC. Amiodarone pulmonary toxicity: recognition and pathogenesis. Part I. Chest 1988; 93: 1067-1075.

22. Martin WJ II, Rosenow EC. Amiodarone pulmonary toxicity: recognition and pathogenesis. Part II. Chest 1988; 93: 1242-1248.

23. Akoun GM, White JP. Treatment-induced respiratory disorders. In: Dukes MNG, ed. Drug-induced Disorders. 1 Edn. Vol. 3. Amsterdam, Elsevier; 1989.

24. Spain RC, Wittlesey D. Respiratory emergencies in patients with cancer. Semin Oncol 1989; 16: 471-489.

25. Anonymous. Drugs that cause pulmonary toxicity. Med Lett Drug Ther 1990; 32: 88-90.

26. Taylor CR. Diagnostic imaging techniques in the evaluation of drug-induced pulmonary disease. Clin Chest Med 1990; 11: 1187-1194.

27. Twohig KJ, Matthay RA. Pulmonary effects of cytotoxic agents other than bleomycin. Clin Chest Med 1990; 31: $31-54$.

28. Walker-Smith G. The histopathology of pulmonary reactions to drugs. Clin Chest Med 1990; 31: 95-118.

29. Massin F, Coudert B, Marot JP, Foucher P, Camus P, Jeannin L. La pneumopathie du méthotrexate. Rev Mal Respir 1990; 7: 5-15.

30. Kuhlman JE. The role of chest computed tomography in the diagnosis of drug-related reactions. $J$ Thoracic Imaging 1991; 6: 52-61.

31. Pietra GG. Pathologic mechanisms of drug-induced lung disorders. J Thoracic Imaging 1991; 6: 1-7.

32. Massin F. Coudert B, Foucher P, et al. La pneumopathie des nitrosourées. Rev Mal Respir 1992; 9: 575-582.

33. Bryant DH. Drug-induced pulmonary disease. Med $J$ Aust 1992; 156: 802-805.

34. Rosenow EC, Myers JL, Swensen SJ, Pisani RJ. Druginduced pulmonary disease: an update. Chest 1992; 102: 239-250.

35. Rosenow EC. Drug-induced pulmonary disease. Dis Month 1994; 40: 253-312.

36. Aronchick JM, Gefter WB. Drug-induced pulmonary disorders. Semin Roentgenol 1995; 30: 18-34.
37. Camus P, Gibson GJ. Adverse pulmonary effects of drugs and radiation. In: Brewis RM, Corrin $\mathrm{B}$, Geddes DM, Gibson GJ, eds. Respiratory Medicine. Vol. 1. Chapter 22. London, W.B. Saunders. 1995; pp. 630-657.

38. Visser LE, Vlug AE, Vanderlei J, Stricker BHC. Cough due to ace inhibitors: a case-control study using automated general practice data. Eur J Clin Pharmacol 1996; 49: 439-444.

39. Pfitzenmeyer P, Meier M, Zuck P, et al. Piroxicaminduced pulmonary infiltrates and eosinophilia. J Rheumatol 1994; 21: 1573-1577.

40. Pfitzenmeyer P, Foucher P, Dennewald G, et al. Pleuropulmonary changes induced by ergoline drugs. Eur Respir J 1996; 9: 1013-1019.

41. Abenhaim L, Moride Y, Brenot F, et al. Appetitesuppressant drugs and the risk of primary pulmonary hypertension. N Engl J Med 1996; 335: 609-616.

42. Kleiger RE, Boxer M, Ingram RE, Harrison DC. Pulmonary hypertension in patients using oral contraceptives. Chest 1976; 69: 143-147.

43. Townend JN, Roberts DH, Jones RL, Davies MK. Fatal pulmonary veno-occlusive disease after use of oral contraceptives. Am Heart J 1992; 124: 1643-1644.

44. Oakley C, Somerville J. Oral contraceptive and progressive pulmonary vascular disease. Lancet 1968; i: 890-893.

45. Lombard JN, Bonnotte B, Maynadié M, et al. Celiprolol pneumonitis. Eur Respir J 1993; 9: 558-591.

46. Popa V. Captopril-related (and induced?) asthma. Am Rev Respir Dis 1987; 136: 999-1000.

47. Benard A, Melloni B, Gosselin B, Bonnaud F, Wallaert B. Perindopril-associated pneumonitis. Eur Respir $J$ 1996; 9: 1314-1316.

48. Berkin KE, Ball SG. Cough and angiotensin-converting enzyme inhibition. $\mathrm{Br}$ Med $J$ 1988; i: 1279-1280.

49. Forslund T, Tohmo H, Weckstrom G, Stenborg M, Jarvinen S. Angio-oedema induced by enalapril. J Intern Med 1995; 238: 179-181.

50. Israili $\mathrm{ZH}$, Hall WD. Cough and angioneurotic edema associated with angiotensin-converting enzyme inhibitor therapy. Ann Intern Med 1992; 117: 234-242.

51. Diem LV, Grilliat JP. Anaphylactic shock induced by paracetamol. Eur J Clin Pharmacol 1990; 38: 389-390.

52. Ellis M, Haydik I, Gillman S, Cummins L, Cairo MS. Immediate adverse reactions to acetaminophen in children: evaluation of histamine release and spirometry. $J$ Pediatr 1989; 114: 654-656.

53. Berrissoul F, Ang-Chin S, Mongin-Charpin D, Payot J, Guibout $P$. Pneumopathie à éosinophiles due au paracétamol: 1er cas de la littérature. Rev Mal Respir 1986; 3: 282.

54. Ho SWC, Beilin LJ. Asthma associated with N-acetylcysteine infusion and paracetamol poisoning: report of two cases. Br Med $J$ 1983; ii: 876.

55. Heffner JE, Sahn SA. Salicylate-induced pulmonary edema: clinical features and prognosis. Ann Intern Med 1981; 95: 405-409.

56. Pelz DM, Lownie SP, Fox AJ, Hutton LC. Symptomatic pulmonary complications from liquid acrylate embolization of brain arteriovenous malformations. Am J Neuroradiol 1995; 16: 19-26.

57. Pusateri DW, Muder RR. Fever, pulmonary infiltrates, and pleural effusion following acyclovir therapy for herpes zoster opththalmicus. Chest 1990; 98: 754-756.

58. Aggarwal A, Farber NE, Warltier DC. Intraoperative bronchospasm caused by adenosine. Anesthesiology 1993; 79: 1132-1135. 
59. Ersoz N, Finestone SC. Adrenaline-induced pulmonary oedema and its treatment: a report of two cases. $\mathrm{Br} \mathrm{J}$ Anaesth 1971; 43: 709-712.

60. Pisani RJ, Rosenow ECI. Pulmonary edema associated with tocolytic therapy. Ann Intern Med 1989; 110: 714-718.

61. Worell JA, O'Donnell DM, Caroll FE, Bruner JP, Coleman JA Jr. Chest case of the day. Case 1: pulmonary edema associated with tocolytic therapy. Am J Roentgenol 1992; 158: $1356-1357$.

62. Worell JA, O'Donnell DM, Caroll FE, Bruner JP, Coleman JA Jr. Chest case of the day. Am J Roentgenol 1992; 158: 898 .

63. Jarzobski J, Ferry J, Wombolt D, Fitch DM, Egan JD. Vasculitis with allopurinol therapy. Am Heart J 1970; 79: 116-121.

64. Aldrich TK, Prezant DJ. Adverse effects of drugs on the respiratory muscles. Clin Chest Med 1990; 11: 177-189.

65. Gurtner HP. Pulmonary hypertension, "plexogenic pulmonary arteriopathy" and the appetite depressant drug aminorex: post or propter? Bull Eur Physiopathol Respir 1979; 15: 897-923.

66. Colgan T, Simon GT, Kay JM, Pugsley SO, Eydt J. Amiodarone pulmonary toxicity. Ultrastruct Pathol 1984; 6: 199-207.

67. Standertskjöld-Nordenstam CG, Wandtke JC, Hood WJ Jr, Zugibe FT, Butler L. Amiodarone pulmonary toxicity: chest radiography and $\mathrm{CT}$ in asymptomatic patients. Chest 1985; 88: 143-145.

68. Clarke B, Ward DE, Honey M. Pneumonitis with pleural and pericardial effusion and neuropathy during amiodarone therapy. Int J Cardiol 1985; 8: 81-88.

69. Gibb P, Melendez LJ. Segmental pulmonary consolidation due to amiodarone. Can Med Assoc J 1986; 134 : 611-613.

70. Manzano Espinosa L, Yebra Bango M, Merino Morales F, L'ados Comange G, de Diego Marin EJ, Durantez Martinez A. Afeccion pulmonar por la amiodarona y anticuerpos antinucleares. Rev Clin Esp 1986; 179: 54-55.

71. Kennedy JI, Myers JL, Plumb VJ, Fulmer JD. Amiodarone pulmonary toxicity: clinical, radiologic and pathologic correlations. Arch Intern Med 1987; 147: 50-55.

72. Myers JL, Kennedy JI, Plumb VJ. Amiodarone lung: pathologic findings in clinically toxic patients. Hum Pathol 1987; 18: 349-354.

73. Dunn M, Glassroth J. Pulmonary complications of amiodarone toxicity. Progr Cardiovasc Dis 1988; 31: 447453.

74. Kuhlman JE, Teigen C, Ren H, Hurban RH, Hutchins GM, Fishman EK. Amiodarone pulmonary toxicity: CT findings in symptomatic patients. Radiology 1990; 177: 121-125.

75. Marshall A, Moore K. Pulmonary disease after amitriptyline overdosage. Br Med J 1973; i: 716-717.

76. Krimerman S, Potasman I, Barak A. Pulmonary reactions associated with the combined use of amphotericin B and leukocyte transfusion. Crit Care Med 1989; 17: 1081.

77. Roncoroni AJ, Corrado C, Besuschio S, Pavlovski S, Narvaiz M. Bronchiolitis obliterans possibly associated with amphotericin B. J Infect Dis 1990; 161: 589.

78. Levine SJ, Walsh TJ, Martinez A, Eichacker PQ, LopezBerestein G, Natanson C. Cardiopulmonary toxicity after liposomal amphotericin B infusion. Ann Intern Med 1991; 114: 664-666.

79. Wright DG, Robichaud KJ, Pizzo PA, Delseroth AB.
Lethal pulmonary reactiosn associated with the combined use of amphotericin B and leukocyte transfusions. N Engl J Med 1981; 304: 1185-1189.

80. Poe RH, Condemi JJ, Weinstein SS, Schuster RJ. Adult respiratory distress syndrome related to ampicillin sensitivity. Chest 1980; 77: 449.

81. Wilsmhurst PT, Webb-Peploe MM. Side-effects of amrinone therapy. Br Heart J 1983; 49: 447-451.

82. Dean NC, Amend WC, Matthay MA. Adult respiratory distress syndrome related to antilymphocyte globulin therapy. Chest 1987; 91: 619-620.

83. Haskell CM, Canellos GP, Leventhal BG, et al. L-asparaginase: therapeutic and toxic effects in patients with neoplastic disease. $N$ Engl J Med 1969; 281: 1028-1034.

84. Shapiro S, Slone D, Lewis GP, Jick H. Fatal drug reactions among medical in-patients. J Am Med Assoc 1971; 216: 467-472.

85. Barbui T, Rodeghiero F, Meli S, Dini E. Fatal pulmonary embolism and antithrombin III deficiency in adult lymphoblastic leukaemia during L-asparaginase therapy. Acta Hematol 1983; 69: 188-191.

86. Evans RB, Ettensohn DB, Fawaz-Estrup F, Lally EV, Kaplan SR. Gold lung: recent developments in pathogenesis, diagnosis, and therapy. Semin Arthr Rheum 1987; 16: 196-205.

87. Morley TF, Komansky HJ, Adelizzi RA, Giudice JC. Pulmonary gold toxicity. Eur J Respir Dis 1984; 65: 627-632.

88. James DW, Whimster WF, Hamilton EBD. Gold lung. Br Med J 1978; i: 1523-1524.

89. Cooke N, Bamji A. Gold lung. Rheum Rehab 1981; 20: 129-135.

90. Ettensohn DB, Roberts NJ, Condemi JJ. Bronchoalveolar lavage in gold lung. Chest 1984; 85: 569-570.

91. Slingerland R, Hoogsteden HC, Adriaansen HJ, van der Kwast TH, Hilvering C. Gold-induced pneumonitis. Respiration 1987; 52: 232-236.

92. Agarwal R, Sharma SK, Malaviya AN. Gold-induced hypersensitivity pneumonitis in a patient with rheumatoid arthritis. Clin Exp Rheumatol 1989; 7: 89-90.

93. Cohen AJ, King TE Jr, Downey GP. Rapidly progressive bronchiolitis obliterans with organizing pneumonia. Am J Respir Crit Care Med 1994; 149: 1670-1675.

94. Albazzaz MK, Harvey JE, Hoffman JN. Alveolitis and haemolytic anemia induced by azapropazone. $\mathrm{Br}$ Med $J$ 1986; 293: 1537-1538.

95. Bedrossian CWM, Sussman J, Conklin RH, Kahan B. Azathioprine-associated interstitial pneumonitis. Am J Clin Pathol 1984; 82: 148-154.

96. LeMense GP, Strange C. Granulomatous pneumonitis following intravesical BCG: What therapy is needed? Chest 1994; 106: 1624-1626.

97. Jimenez I, Anton E, Picans I, Jerez J. Betahistine-induced bronchospasm. Allergy 1996; 51: 185-188.

98. Vasilomanolakis EC, Goldberg NM. Bepridil-induced pulmonary fibrosis. Am Heart J 1993; 126: 1016-1017.

99. Balikian JP, Jochelson MS, Bauer KA, et al. Pulmonary complications of chemotherapy regimens containing bleomycin. Am J Roentgenol 1982; 139: 455-461.

100. Rose AG. Pulmonary veno-occlusive disease due to bleomycin therapy for lymphoma. S Africa Med J 1983; 64: 636-638.

101. Mayr B, Bauer WM, Clemm C, Hartenstein R. Differentialdiagnostische Probleme bei der Abgrenzung von Lungeninfiltraten nach Bleomycintherapie gegenüber Metastasen. Fortschr Röntgenstr 1984; 141: 409-414.

102. Leeser JE, Carr D. Fatal pneumothorax following bleomycin 
and other cytotoxic drugs. Cancer Treat Rep 1985; 69: 344-345.

103. Rimmer MJ, Dixon AK, Flower CDR, Sikora K. Bleomycin lung: computed tomographic observations. Br J Radiol 1985; 58: 1041-1045.

104. Yousem SA, Lifson JD, Colby TV. Chemotherapyinduced eosinophilic pneumonia: relation to bleomycin. Chest 1985; 88: 103-106.

105. Doll DC. Fatal pneumothorax associated with bleomycin-induced pulmonary fibrosis. Cancer Chemother Pharmacol 1986; 17: 294-295.

106. Jules-Elysee K, White DA. Bleomycin-induced pulmonary toxicity. Clin Chest Med 1990; 11: 1-20.

107. Zitnik RJ. Drug-induced lung disease: cancer chemotherapy agents. J Respir Dis 1995; 16: 855-865.

108. De Wolf AM, Van den Berg BW, Hoffman HJ, Van Zundert AA. Pulmonary dysfunction during one-lung ventilation caused by HLA-specific antibodies against leukocytes. Anesth Analg 1987; 66: 463-467.

109. Dubois M, Lotze MT, Diamond WJ, Kim YD, Flye MW, Macnamara TE. Pulmonary shunting during leukoagglutinin-induced noncardiac pulmonary edema. $J$ Am Med Assoc 1980; 244: 2186-2189.

110. Eastlund T, McGrath PC, Britten A, Propp R. Fatal pulmonary transfusion reaction to plasma containing donor HLA antibody. Vox Sang 1989; 57: 63-66.

111. Popovsky MA, Moore SB. Diagnostic and pathogenetic considerations in transfusion-related acute lung injury. Transfusion 1985; 25: 573-577.

112. Popovsky MA, Abel MD, Moore SB. Transfusion-related acute lung injury associated with passive transfer of antileukocyte antibodies. Am Rev Respir Dis 1983; 128: 185-189.

113. Levy GJ, Shabot MM, Hart ME, Mya WW, Goldfinger D. Transfusion-associated noncardiogenic pulmonary edema. Transfusion 1986; 26: 278-281.

114. Ebert JP, Grimes B, Niemann KMW. Respiratory failure secondary to homologous blood transfusion. Anesthesiology 1985; 63: 104-106.

115. Ogawa H, Fujimura M, Heki U, Kitagawa M, Matsuda $\mathrm{T}$. Eosinophilic bronchitis presenting with only severe dry cough due to bucillamine. Respir Med 1995; 89: 219-221.

116. Cooke CR, Hyland JW. Pathological calcifications of the lungs following intravenous administration of calcium. Am J Med 1960; 29: 363-368.

117. Mani TM, Lalleland D, Coreone S, Mauriat D. Metastatic pulmonary calcifications after cardiac surgery in children. Radiology 1990; 174: 463-467.

118. Lee T, Cochrane GM. Pulmonary eosinophilia and asthma associated with carbamazepine. Br Med J 1981; i: 440.

119. Lewis IJ, Rosenbloom L. Glandular fever-like syndrome, pulmonary eosinophilia and asthma associated with carbamazepine. Postgrad Med J 1982; 58: 100-101.

120. Kitson GE, Wauchob TD. Pulmonary oedema following carbamazepine overdose. Anaesthesia 1988; 43: 967-969.

121. Schatz PL, Mesologites D, Hyun J, Walker Smith GJ, Lahiri B. Captopril-induced hypersensitivity lung disease. Chest 1989; 95: 685-687.

122. Kidney JC, O'Halloran DJ, FitzGerald MX. Captopril and lymphocytic alveolitis. $\mathrm{Br}$ Med J 1989; ii: 981.

123. Takahashi N, Aizawa H, Takata S, et al. Acute interstitial pneumonitis induced by carbamazepine. Eur Respir $J$ 1993; 6: 1409-1411.

124. King GG, Barnes DJ, Hayes MJ. Carbamazepine-induced pneumonitis. Med J Aust 1994; 160: 126-127.
125. De Vriese ASP, Philippe J, Van Renterghem DM, De Cuyper CA, Hindryckx EGJ, Louagie A. Carbamazepine hypersensitivity syndrome: report of 4 cases and review of the literature. Medicine (Baltimore) 1995; 74: 144150.

126. Selker RG, Jacobs SA, Moore PB, et al. 1,3-bis(2chloroethyl)-1-nitrosourea (BCNU)-induced pulmonary fibrosis. Neurosurgery 1980; 7: 560-565.

127. Grinblat J, Mechlis S, Lewitus Z. Organizing pneumonia-like process: an unusual observation in steroid responsive cases with features of chronic interstitial pneumonia. Chest 1981; 80: 259-263.

128. Dreis DF, Winterbauer RH, van Norman GA, Sullivan SL, Hammar SP. Cephalosporin-induced interstitial pneumonitis. Chest 1984; 86: 138-140.

129. Bochner BS, Lichtenstein LM. Anaphylaxis. $N$ Engl J Med 1991; 324: 1785-1790.

130. Crestani B, Jaccard A, Israel-Biet D, Couderc LJ, Frija J, Clauvel JP. Chlorambucil-associated pneumonitis. Chest 1994; 105: 634-636.

131. Cooper JAD, White DA, Matthay RA. Drug-induced pulmonary disease. Part II. Noncytotoxic drugs. Am Rev Respir Dis 1986; 133: 488-805.

132. Roche-Bayard P, Rossi R, Mann JM, Cordier JF, Delahaye JP. Left pulmonary artery thrombosis in chlorpromazineinduced lupus. Chest 1990; 98: 1545.

133. Bell RJM. Pulmonary infiltration with eosinophils caused by chlorpropamide. Lancet 1964; i: 1249-1250.

134. Peters B, Pinching AJ. Fatal anaphylaxis associated with ciprofloxacin in a patient with AIDS-related complex. Br Med J 1989; i: 605.

135. Finet JF, Similowski T, Derenne JP, Le Charpentier Y. Surcharge pulmonaire réversible à la clofazimine (Lamprene $\left.{ }^{\circledR}\right)$ chez des sujets VIH+. Ann Pathol 1995; 15: 224-225.

136. Hendrickson RM, Simpson F. Clofibrate and eosinophilic pneumonia. J Am Med Assoc 1982; 247: 3082.

137. Zosmer A, Katz Z, Lancet M, Konichezky S, SchwartzShoham Z. Adult respiratory distress syndrome complicating ovarian hyperstimulation syndrome. Fertil Steril 1987; 47: 524-526.

138. Caliandro R, Labalme MJ, Pérol M, Nicollet B, Guérin JC. Pleurésie aiguë secondaire à une hyperstimulation ovarienne après fécondation in vitro. Rev Mal Respir 1996; 13: 68-70.

139. Witman G, Davis R. A lupus erythematosus syndrome induced by clonidine hydrochloride. Rhode Isl Med J 1981; 64: 147-150.

140. Hill RN, Spragg RG, Wedel MK, Moser KM. Adult respiratory distress syndrome associated with colchicine intoxication. Ann Intern Med 1975; 83: 523-524.

141. Pemberton LB. Shock lung with massive tracheal loss of plasma. J Am Med Assoc 1977; 237: 2511-2513.

142. Huang TY, Peterson GH. Pulmonary edema and iododerma induced by potassium iodide in the treatment of asthma. Ann Allergy 1981; 46: 264-266.

143. Jennings CA, Deveikis J, Azumi N, Yeager H Jr. Eosinophilic pneumonia associated with reaction to radiographic contrast medium. South Med J 1991; 84: 92-95.

144. Lang DM, Alpern MB, Visintainer PF, Smith ST. Increased risk for anaphylactoid reaction from contrast media in patients on $\beta$-adrenergic blockers or with asthma. Ann Intern Med 1991; 115: 270-276.

145. Goldsmith SR, Steinberg P. Noncardiogenic pulmonary edema induced by nonionic low-osmolality radiographic contrast media. J Allergy Clin Immunol 1995; 96: 698699.

146. Guérin JC, Kofman J, Chevalier JP, Perrin-Fayolle M, 
Kalb JC. Pneumopathie d'hypersensibilité au sulfamethoxazole-trimethoprime. Rev Fr Mal Respir 1980; 8: 406-407.

147. Holdcroft CJ, Ellison RTI. Trimethoprim-sulfamethoxazole reaction simulating Pneumocystis carinii pneumonia. AIDS 1991; 5: 1029-1032.

148. Sheffer AL, Rocklin RE, Goetzl EJ. Immunologic components of hypersensitivity reactions to cromolyn sodium. N Engl J Med 1975; 293: 1220-1224.

149. Salles G, Vial T, Archimbaud E. Anaphylactoid reaction with bronchospasm following intravenous cyclophosphamide administration. Ann Hematol 1991; 62: 74-75.

150. Burke DA, Stoddart JC, Ward MK, Simpson CGB. Fatal pulmonary fibrosis occurring during treatment with cyclophosphamide. Br Med J 1982; ii: 696.

151. Gould VE, Miller J. Sclerosing alveolitis induced by cyclophosphamide. Am J Pathol 1975; 81: 513-530.

152. Usui Y, Aida H, Kimula Y, et al. A case of cyclophosphamide-induced interstitial pneumonitis diagnosed by bronchoalveolar lavage. Respiration 1992; 59: 125-128.

153. Maxwell I. Reversible pulmonary edema following cyclophosphamide treatment. J Am Med Assoc 1974; 229: $137-138$.

154. Schaap N, Raymakers R, Schattenberg A, Ottevanger JP, de Witte T. Massive pleural effusion attributed to high-dose cyclophosphamide during conditioning for BMT. Bone Marrow Transplantation 1996; 18: 247-248.

155. Powell-Jackson PR, Carmichael FJL, Calne RY, Williams R. Adult respiratory distress syndrome and convulsions associated with administration of cyclosporine in liver transplant recipients. Transplantation 1984; 38: 341343.

156. Carbone L, Appel GB, Benvenisty AI, Cohen DJ, Kunis CL, Hardy MA. Adult respiratory distress syndrome associated with oral cyclosporine. Transplantation 1987; 43: 767-768.

157. Blaauw AAM, Leunissen KML, Cheriex EC, Wolters J, Kootstra G, van Hooff JP. Disappearance of pulmonary leak syndrome when intravenous cyclosporine is replaced by oral cyclosporine. Transplantation 1987; 43: 758-759.

158. Gould K, Freeman R, Odom NJ, Locke TJ, McGregor CGA. Pulmonary "cyclosporinoma" mimicking infection after heart transplantation. J Heart Transplant 1987; 6: 375-377.

159. Harris KM, Schwartz ML, Slasky BS, Nalesnik M, Makowka L. Posttransplantation cyclosporine-induced lymphoproliferative disorders: clinical and radiologic manifestations. Radiology 1987; 162: 697-700.

160. Haupt HM, Hutchins GM, Moore GW. Ara-C lung: noncardiogenic pulmonary edema complicating cytosine arabinoside therapy of leukaemia. Am J Med 1981; 70: 256-261.

161. Andersson BS, Cogan BM, Keating MJ, Estey EH, McCreadie KB, Freireich EJ. Subacute pulmonary failure complicating therapy with high dose Ara-C in acute leukemia. Cancer 1985; 56: 2181-2184.

162. Jehn U, Göldel N, Rienmüller R, Wilmanns W. Noncardiogenic pulmonary edema complicating intermediate and high-dose Ara-C treatment for relapsed acute leukemia. Med Oncol Tumor Pharmacother 1988; 5: 41-47.

163. Andersson BS, Luna BS, Yee C, Hui KK, Keating MJ, McCreadie KB. Fatal pulmonary failure complicating high-dose cytosine arabinoside therapy in acute leukemia. Cancer 1990; 65: 1079-1084.

164. Motomura S, Fujisawa S, Fujimaki K, Mohri H, Okubo
T. Interstitial pneumonia induced by combination therapy with low-dose cytarabine and granulocyte colonystimulating factor. Am J Hematol 1995; 49: 364.

165. Miller DH, Haas LF. Pneumonitis, pleural effusion and pericarditis following treatment with dantrolene. J Neurol Neurosurg Psychiatr 1984; 47: 553-554.

166. Petusevsky ML, Faling LJ, Rocklin RE, et al. Pleuropericardial reaction to treatment with dantrolene. $J$ Am Med Assoc 1979; 242: 2772-2774.

167. Gallant JE, Hoehn-Saric E, Smith MD. Respiratory insufficiency from dapsone-induced methemoglobinemia. AIDS 1991; 5: 1392-1393.

168. Freedman MH, Grisaru D, Olivieri N, McLusky I, Thorner PS. Pulmonary syndrome in patients with thalassemia major receiving intravenous deferoxamine infusions. $\mathrm{Am}$ $J$ Dis Child 1990; 144: 565-569.

169. Cianciulli P. Pulmonary embolism and intravenous highdose desferrioxamine. Haematologica 1992; 77: 368- 369.

170. Freedman GS, Lofgen SB, Kligerman MM. Radiationinduced changes in pulmonary perfusion. Radiology 1974; 112: 435-437.

171. Castriota Scanderberg A, Izzi GC, Butturini A, Benaglia G. Pulmonary syndrome and intravenous high-dose desferrioxamine. Lancet 1990; ii: 1511.

172. Panuska JR, King TR, Korenblat PE, Wedner HJ. Hypersensitivity reaction to desipramine. J Allergy Clin Immunol 1987; 80: 18-23.

173. Mutnick A, Schneiweiss F. Desipramine-induced pulmonary interstitial eosinophilia. Drug Intell Clin Pharm 1982; 16: 966-967.

174. Kraus RP, Clarke RJ, Remick RA. Massive eosinophilic reaction to desipramine in conjunction with pneumonia. Can J Psychiatr 1984; 29: 142-144.

175. Desai DK, Moodley J, Naidoo DP, Bhorat I. Cardiac abnormalities in pulmonary oedema associated with hypertensive crises in pregnancy. Br J Obstet Gynaecol 1996; 103: 523-528.

176. Pouwels HMM, Smeets JLRM, Cheriex EC, Wouters EFM. Pulmonary hypertension and fenfluramine. Eur Respir J 1990; 3: 606-607.

177. Braun D, Tréchot P, Netter P, Danloy V, Anthoine D, Vaillant G. Recurrent interstitial pneumonitis and dexfenfluramine. Chest 1993; 103: 1927.

178. Brenot F, Hervé P, Petitpretz P, Parent F, Duroux P, Simonneau G. Primary pulmonary hypertension and fenfluramine use. Br Heart J 1993; 70: 537-541.

179. Khalil H, Molinari E, Stoller JK. Diclofenac (Voltaren)induced eosinophilic pneumonitis. Arch Intern Med 1993; 153: 1649-1652.

180. Szczeklik A, Gryglewski RJ, Czerniawska-Mysik G, Pieton R. Asthmatic attacks induced in aspirin-sensitive patients by diclofenac and naproxen. Br Med J 1977; ii: $231-232$.

181. Bass BH. Hydralazine lung. Thorax 1981; 36: 695-696.

182. Carey RM, Coleman M, Feder A. Pericardial tamponade: a major presenting manifestation of hydralazineinduced lupus syndrome. Am J Med 1973; 54: 84-87.

183. Richards FM, Fulkerson WJ Jr. Constrictive pericarditis due to hydralazine-induced lupus erythematosus. Am J Med 1990; 88: 56-59.

184. Ripe E, Nilsson BS. Pulmonary inhalation during dihydralazine treatment in a slow isoniazid-inactivator. Scand J Respir Dis 1972; 53: 56-63.

185. Short AK, Lockwood CM. Antigen specificity in hydralazine associated ANCA positive systemic vasculitis. QJM 1995; 88: 775-783.

186. Curt GA, Kelley JA, Fine RL, et al. A phase I and 
pharmacokinetic study of dihydro-5-azacytidine (NSC 264880). Cancer Res 1985; 45: 3359-3363.

187. Humbert VH, Munn NJ, Hawkins RF. Noncardiogenic pulmonary edema complicating massive diltiazem overdose. Chest 1991; 99: 258-260.

188. Diebold J, James J-M, Dao C, et al. Hyperplasie lymphoïde immunoblastique pseudo-tumorale par hypersensibilité médicamenteuse. Arch Anat Cytol Pathol 1976; 24: 189-194.

189. Zupnick HM, Brown LK, Miller A, Moros DA. Respiratory dysfunction due to L-dopa therapy for parkinsonism: diagnosis using serial pulmonary function tests and respiratory inductive plethysmography. Am J Med 1990; 89: 109-114.

190. McEwan SR, Clark RA, Guthrie W. Dothiepin and fatal fibrosing alveolitis. Lancet 1986; i: 970.

191. Veale D, Gilmartin JJ. Acute alveolitis associated with dothiepin treatment. Br Med J 1985; i: 606.

192. Giannocarro PJ, Wallace GJ, Higginson LA, Williams WL. Fatal angioedema associated with enalapril. Can J Cardiol 1989; 5: 335-336.

193. Gianos ME, Klaustermeyer WB, Kurohara M, Tarnasky P, Gordon E. Enalapril-induced angioedema. Am J Emerg Med 1990; 8: 124-126.

194. Talwar KK, Kothari SS, Bhatia ML. Bronchospasm following ergometrine testing for coronary spasm. Am Heart $J$ 1985; 109: 1415.

195. Abramov LA, Yust IC, Fierstater EM, Vardinon NE. Acute respiratory distress caused by erythromycin hypersensitivity. Arch Intern Med 1978; 138: 1156-1158.

196. Wong PC, Yew WW, Wong CF, Choi HY. Ethambutolinduced pulmonary infiltrates with eosinophilia and skin involvement. Eur Respir J 1995; 8: 866-868.

197. Miller KS, Sahn SA. Bilateral exudative pleural effusions following intravenous ethchlorvynol administration. Chest 1989; 95: 464-465.

198. Glaholm J, Repetto L, Yarnold JR, et al. Carboplatin (JM8), etoposide (VP16) and thoracic irradiation for small cell lung cancer: an evaluation of lung toxicity. Radiother Oncol 1988; 12: 31-37.

199. Kellie JS, Crist WM, Pui C-H, et al. Hypersensitivity reactions to epipodophyllotoxins in children with acute lymphoblastic leukemia. Cancer 1991; 67: 1070-1075.

200. Yano S, Shimada K. Vasospastic angina after chemotherapy with carboplatin and etoposide in a patient with lung cancer. Jap Circ J 1996; 60: 185-188.

201. Burton GH. Rash and pulmonary eosinophilia associated with fenbrufen. BMJ 1990; i: 82-83.

202. Goodwin SD, Glenny RW. Nonsteroidal anti-inflammatory drug-associated pulmonary infiltrates with eosinophilia. Arch Intern Med 1992; 152: 1521-1524.

203. Barandi F, Quenzer F, Rapp K. Pulmonary hypersensitivity to nalfon. Ann Allergy 1986; 57: 205-207.

204. Gentzkow GD, Sullivan JY. Extracardiac adverse effect of flecainide. Am J Cardiol 1984; 53: 101B-105B.

205. Wong MK, Bjarnason GA, Hrushesky WJ, Webster P, Morava-Protzner I, Towers M. Steroid-responsive interstitial lung disease in patients receiving 2'-deoxy-5-fluorouridine-infusion chemotherapy. Cancer 1995; 75: $2558-2564$

206. Cervantes F, Salgado C, Montserrat E, Rozman C. Fludarabine for prolymphocytic leukaemia and risk of interstitial pneumonitis. Lancet 1990; ii: 1130.

207. Kane GC, McMichael AJ, Patrick H, Erslev AJ. Pulmonary toxicity and acute respiratory failure associated with fludarabine monophosphate. Respir Med 1992; 86: 261-263.
208. Bosso JV, Creighton D, Stevenson DD. Flurbiprofen (a NSAID) cross-sensitivity in an aspirin-sensitive asthmatic patient. Chest 1992; 101: 856-858.

209. Bass SP, Colebatch HJH. Fluoxetine-induced lung damage. Med J Aust 1992; 156: 364-365.

210. Davies LP. Fluoxetine-induced lung damage. Med J Aust 1992; 156: 740.

211. de Kerviler E, Trédaniel J, Revlon G, et al. Fluoxetininduced pulmonary granulomatosis. Eur Respir J 1996; 9: 615-617.

212. Gonzalez-Rohti RJ, Zander DS, Ros PR. Fluoxetine hydrochloride (Prozac)-induced pulmonary disease. Chest 1995; 107: 1763-1765.

213. Cortez LM, Pankey GA. Acute pulmonary hypersensitivity to furazolidone. Am Rev Respir Dis 1972; 105: $823-826$.

214. Gheysens B, van Mieghem W. Pulmonary infiltrates with eosinophilia due to glafenine. Eur J Respir Dis 1984; 65: 456-459.

215. Clarke BF, Campbell IW, Ewing DJ, Beveridge GW, MacDonald MK. Generalized hypersensitivity reaction and visceral arteritis with fatal outcome during glibenclamide therapy. Diabetes 1974; 23: 739-742.

216. Katoh M, Shikoshi K, Takada M, et al. Development of interstitial pneumonitis during treatment with granulocyte colony-stimulating factor. Ann Hematol 1993; 67: 201-202.

217. Lei KIK, Leung WT, Johnson PJ. Serious pulmonary complications in patients receiving recombinant granulocyte colony-stimulating factor during BACOP chemotherapy for aggressive non-Hodgkin's lymphoma. $\mathrm{Br} \mathrm{J}$ Cancer 1994; 70: 1009-1013.

218. Seebach J, Speich R, Fehr J, Tuchschmid P, Russi E. GM-CSF-induced acute eosinophilic pneumonia. $\mathrm{Br} \mathrm{J}$ Haematol 1995; 90: 963-965.

219. Bastion Y, Reyes F, Bosly A, et al. Possible toxicity with the association of G-CSF and bleomycin. Lancet 1994; i: 1221-1222.

220. Iki S, Yoshinaga K, Ohbayashi Y, Urabe A. Cytotoxic drug-induced pneumonia and possible augmentation by G-CSF: clinical attention. Ann Hematol 1993; 66: 217218.

221. Philippe B, Couderc LJ, Balloul-Delclaux E, Janvier M, Caubarrère I. Pulmonary toxicity of chemotherapy and GM-CSF. Respir Med 1994; 88: 715.

222. Mahutte CK, Nakasato SK, Light RW. Haloperidol and sudden death due to pulmonary edema. Arch Intern Med 1982; 142: 1951-1952.

223. Asimacopoulos PJ, Athanasiadis, McCarthy JJ, Shade RJ, Teague RB. Can heparin cause adult respiratory distress syndrome by a similar mechanism as heparin-associated thrombocytopenia? Chest 1994; 105: 1266-1268.

224. Perry HM Jr, O'Neal RM, Thomas WA. Pulmonary disease following chronic chemical ganglionic blockade. Am J Med 1957; 22: 37-50.

225. Nässberger L, Sjöholm AG, Jonsson H, Sturfelt G, Akesson A. Autoantibodies against neutrophil cytoplasm components in systemic lupus erythematosus and in hydralazine-induced lupus. Clin Exp Immunol 1990; 81: 380-383.

226. Weinstein J. Hypocomplementemia in hydralazine-associated systemic lupus erythematosus. Am J Med 1978; 65: 553-556.

227. Dawkins KD, Burke CM, Billingham ME, Jamieson SW. Primary pulmonary hypertension and pregnancy. Chest 1986; 89: 383-388.

228. Steinberg AD. Pulmonary edema following ingestion 
of hydrochlorothiazide. J Am Med Assoc 1968; 204:167168.

229. Bell RT, Lippmann M. Hydrochlorothiazide-induced pulmonary edema. Arch Intern Med 1979; 139: 817-819.

230. Carlson RW, Schaeffer RC, Puri VK, Brennan AP, Weil $\mathrm{MH}$. Hypovolemia and permeability pulmonary oedema associated with anaphylaxis. Crit Care Med 1981; 9: 883-885.

231. Dorn MR, Walker BK. Noncardiogenic pulmonary edema associated with hydrochlorothiazide therapy. Chest 1981; 79: 482-483.

232. Watrigant Y, Wallaert B, Ramon P, Gosselin B, Peng $\mathrm{W}$, Tonnel AB. Pneumopathie à l'hydrochlorothiazide d'évolution subaigue: étude cytologique du lavage broncho-alvéolaire. Rev Mal Respir 1986; 4: 227-229.

233. Bowden FJ. Noncardiogenic pulmonary oedema after ingestion of chlorothiazide. Br Med J 1989; i: 605.

234. Biron P, Dessureault J, Napke E. Acute allergic interstitial pneumonitis induced by hydrochlorothiazide. Can Med Assoc J 1991; 145: 28-34.

235. Banzet ML, David M, Bechtel P. Pneumopathie interstitielle à l'hydroxyquinoléine. Therapie 1986; 41: 315318.

236. Hennemann B, Bross KJ, Reichle A, Andreesen R. Acute alveolitis induced by hydroxyurea in a patient with myeloproliferative syndrome. Ann Hematol 1993; 67: 133134.

237. Chetty KG, Ramirez MM, Mahutte CK. Drug-induced pulmonary edema in a patient infected with human immunodeficiency syndrome. Chest 1993; 104: 967-969.

238. Schooley RT, Wagley PF, Lietman PS. Edema associated with ibuprofen therapy. J Am Med Assoc 1977; 237: 1716-1717.

239. Baker WJ, Fistel SJ, Jones RV, Weiss RB. Interstitial pneumonitis associated with ifosfamide therapy. Cancer 1990; 65: 2217-2221.

240. Cutler NR, Anderson DJ. Proven asymptomatic eosinophilia with imipramine. Am J Psychiatry 1977; 134: 1296-1297.

241. Rault R, Piraino B, Johnston GR, Oral A. Pulmonary and renal toxicity of intravenous immunoglobulin. Clin Nephrol 1991; 36: 83-86.

242. Kamisako T, Adachi Y, Chihara J, Yamamoto T. Interstitial pneumonitis and interferon-alpha. Br Med J 1993; i: 896.

243. Chin K, Tabata C, Satake N, Nagai S, Moriyasu F, Kuno K. Pneumonitis associated with natural and recombinant interferon alpha therapy for chronic hepatitis C. Chest 1994; 105: 939-941.

244. Ogata K, Koga T, Yagawa K. Interferon-related bronchiolitis obliterans organizing pneumonia. Chest 1994; 106: 612-613.

245. Lustman F, Salhadin A, Nouwynck C, Hanson B. Pneumonie interstitielle à la suite d'un traitement par interféron-alpha. Presse Méd 1995; 24: 1910.

246. Vogelzang NJ, Stenlund R. Residual pulmonary nodules after combination chemotherapy of testicular cancer. Radiology 1983; 146: 195-197.

247. Mann H, Ward JH, Samlowski WE. Vascular leak syndrome associated with interleukin-2: chest radiographic manifestations. Radiology 1990; 176: 191-194.

248. Glauser FL, DeBlois G, Bechard D, Fowler AA, Merchant R, Fairman RP. Cardiopulmonary toxicity of adoptive immunotherapy. Am J Med Sci 1988; 296: 406-412.

249. Kalinowski SZ, Lloyd TW, Moyes EN. Complications in the chemotherapy of tuberculosis. Am Rev Respir Dis 1961; 83: 359-371.
250. Miyai M, Tsubota T, Asano K. Isoniazid-induced interstitial pneumonia. Respir Med 1989; 83: 517-519.

251. Perreau P, Fresneau M, Boumard B, Le Brizaut Y. L'infiltrat pulmonaire avec éosinophilie au cours du traitement par l'isoniazide. Presse Méd 1955; 63: 1454-1456.

252. Fraimow W, Wallace $S$, Lewis $P$, Greening RR, Cathcart RT. Changes in pulmonary function due to lymphography. Radiology 1965; 85: 231-241.

253. Oliviero G, Constans P, Caby I, de Rohan-Chabot P, Lacherade JC. Pneumopathie induite par l'isotretinoïne. Rev Mal Respir 1995; 12: 631-633.

254. Sabroe RA, Staughton RCD, Bunker CB. Bronchospasm induced by isotretinoin. $\mathrm{Br}$ Med $J$ 1996; i: 886.

255. Günther J, Lode H, Raffenberg M, Schaberg T. Development of pleural and pericardial effusions during itraconazole therapy of pulmonary aspergillosis. Eur J Clin Microbiol Infect Dis 1993; 12: 723-724.

256. Vacher D, Aumaitre O, Mignot P, Lavarenne J, Molina C. Alvéolite allergique médicamenteuse et syndrome de Parsonnage et Turner chez un malade taité par labetalol. Presse Méd 1987; 16: 539-540.

257. Kheir A, Chabot F, Delorme N, Polu JM. Pneumopathie fibrosante induite par le labétalol. Rev Pneumol Clin 1996; 52: 33-35

258. Gamelin L, Jeanson S, Chenue F, et al. Acute respiratory distress syndrome secondary to leuproprelin therapy. Thérapie 1995; 50: 587-588.

259. Howard JJ, Mohsenifar Z, Simons SM. Adult respiratory distress syndrome following administration of lidocaine. Chest 1982; 81: 644-645.

260. Ulmer JL, Garvey MJ. Fatal angioedema associated with lisinopril. Ann Pharmacother 1992; 26: 1245-1246.

261. Bhatt MH, Keenan SP, Fleetham JA, Calne DB. Pleuropulmonary disease associated with dopamine agonist therapy. Ann Neurol 1991; 30: 613-616.

262. Ii T, Doutsu Y, Ashitani J, et al. A case of loxoprofeninduced pulmonary eosinophilia. Jpn Thorac Dis 1992; 30: 926-929.

263. Watanabe T, Sakata M, Shimabukuro R, et al. Loxoprofen: another NSAID associated with acute asthmatic death. Clin Toxicol 1993; 31: 333-340.

264. Margolis MT, Thoen LD, Mercer LJ, Keith LG. Hemothorax after Lupron therapy of a patient with pleural endometriosis: a case report and literature review. Int J Fertil Menopaus Stud 1996; 41: 53-55.

265. Salmeron S, Brenot F, Rain B, et al. Maprotiline and pulmonary alveolitis. Ann Intern Med 1988; 109: 758-759.

266. Rosketh R, Storstein O. Pulmonary complications during mecamylamine therapy. Acta Med Scand 1960; 167: 23-27.

267. De Greve J, Warson F, Deleu D, Storme G. Fatal pulmonary toxicity by the association of radiotherapy and medroxyprogesterone acetate. Cancer 1985; 56: 2434-2436.

268. Major PP, Laurin S, Bettez P. Pulmonary fibrosis following therapy with melphalan: report of two cases. Can Med Assoc J 1980; 123: 197-202.

269. Westerfield BT, Michalski JP, McCombs C, Light RW. Reversible melphalan-induced lung damage. Am J Med 1980; 68: 767-771.

270. Codling BW, Chakera TMH. Pulmonary fibrosis following therapy with melphalan for multiple myeloma. J Clin Pathol 1972; 25: 668-673.

271. Manigand G, Pointud P, Taillandier J. Hypersensibilité au melphalan; allergie croisée avec le cyclophosphamide. Presse Méd 1981; 10: 2445.

272. Rodman T, Fraimow W, Myerson RM. Löffler's syndrome: report of a case associated with administration 
of mephenesin carbamate (Tolseram). Ann Intern Med 1958; 48: 668-674.

273. Cooper JAD, White DA, Matthay RA. Drug-induced pulmonary disease. Part I. Cytotoxic drugs. Am Rev Respir Dis 1986; 133: 321-340.

274. Camus P, Piard F, Ashcroft T, Gal AA, Colby TV. The lung in inflammatory bowel disease. Medicine (Baltimore) 1993; 72: 151-183.

275. Dombret MC, Saraux JL, Fagon JY, Smiejan JM, Thibault P. Pneumopathies à la métapramine. Presse Méd 1988; 17: 389 .

276. Frand UI, Shim CS, Williams MH. Methadone-induced pulmonary edema. Ann Intern Med 1972; 76: 975-979.

277. Sieniewicz DJ, Nidecker AC. Conglomerate pulmonary disease: a form of talcosis in intravenous methadone abusers. Am J Roentgenol 1982; 135: 697-702.

278. Sostman HD, Matthay RA, Putman CE, Walker Smith GJ. Methotrexate-induced pneumonitis. Medicine (Baltimore) 1976; 55: 371-388.

279. Weiss JR, Bruno S. Hypersensitivity reactions to cancer chemotherapeutic agents. Ann Intern Med 1981; 94: 66-72.

280. Barrera P, Laan RFJM, van Riel PLCM, Dekhuijzen PNR, Boerbooms ATH, van de Putte LBA. Methotrexaterelated pulmonary complications in rheumatoid arthritis. Ann Rheum Dis 1994; 53: 434-439.

281. Renaudier P, Chabot F, Ferry R, et al. Pneumopathies du méthotrexate survenant dans le cadre du traitement des lymphomes non hodkiniens par le protocole $\mathrm{m}$ BACOD. Bull Cancer 1995; 82: 149-154.

282. Nordstrom DM, West SG, Rubin RL. Methyldopa-induced systemic lupus erythematosus. Arthr Rheum 1989; 32: 205-208.

283. Graham RG. Cardiac and pulmonary fibrosis during methysergide therapy for headache. Am J Med Sci 1967; 254: $1-12$

284. Meeran MK, Ahmed AH, Parsons FM, Anderson CK Constrictive pericarditis due to methysergide therapy. $S$ Africa Med J 1976; 50: 1595-1597.

285. Chung M, Chetty KG, Jerome D. Metoclopramide and asthma. Ann Intern Med 1985; 103: 809.

286. Raffy O, Sleiman C, Vachiery F, et al. Refractory hypoxemia during liver cirrhosis: hepatopulmonary syndrome or "primary" pulmonary hypertension? Am J Respir Crit Care Med 1996; 153: 1169-1171.

287. Kristenson M, Fryden A. Pneumonitis caused by metronidazole. J Am Med Assoc 1988; 260: 184.

288. Fainstein V, Bodey GP. Cardiorespiratory toxicity due to miconazole. Ann Intern Med 1980; 93: 432-433.

289. Cellerin L, Canfrere I, Ordronneau J, Chailleux E. Pneumopathie aiguë à éosinophiles induite par la minocycline. Rev Pneumol Clin 1994; 50: 325-328.

290. Kaufman D, Pichler W, Beer JH. Severe episode of high fever with rash, lymphadenopathy, neutropenia, and eosinophilia after minocycline therapy for acne. Arch Intern Med 1994; 154: 1983-1984.

291. Sitbon O, Bidel N, Dussopt C, et al. Minocycline pneumonitis and eosinophilia: a report on 8 patients. Arch Intern Med 1994; 154: 1633-1640.

292. Bando T, Fujimura M, Noda Y, Hirose J, Ohta G, Matsuda T. Minocycline-induced pneumonitis with bilateral hilar lymphadenopathy and pleural effusion. Intern Med 1994; 33: $177-179$.

293. Kameya T, Morita T, Tanaka I, et al. A case of minocycline-induced hypersensitivity pneumonitis with radiographic finding of multiple nodules. Nippon Naika Gakkai Zasshi 1994; 83: 1991-1992.
294. Dykhuizen RS, Legge JS. Minocycline-induced pulmonary eosinophilia. Respir Med 1995; 89: 61-62.

295. Piperno D, Donné C, Loire R, Cordier JF. Bronchiolitis obliterans organizing pneumonia associated with minocycline therapy: a possible cause. Eur Respir J 1995; 8: 1018-1020.

296. Orwoll ES, Kiessling P, Patterson R. Interstitial pneumonia from mitomycin. Ann Intern Med 1978; 89: 352-355.

297. Buzdar AU, Legha SS, Luna MA, Tashima CK, Hortobagyi GN, Blumenschein GR. Pulmonary toxicity of mitomycin. Cancer 1980; 45: 236-244.

298. Chang AYC, Kuebler JP, Pandya KJ, Israel RH, Marshall $\mathrm{BC}$, Tormey DC. Pulmonary toxicity induced by mitomycin $\mathrm{C}$ is highly responsive to glucocorticoids. Cancer 1986; 57: 2285-2290.

299. Hoelzer KL, Harrison BR, Luedcke SW, Luedcke DW. Vinblastine-associated pulmonary toxicity in patients receiving combination therapy with mitomycin and cisplatin. Drug Intell Clin Pharm 1986; 20: 287-289.

300. McCarthy JT, Staats BA. Pulmonary hypertension, hemolytic anemia, and renal failure: a mitomycin-associated syndrome. Chest 1986; 89: 608-611.

301. Sheldon R, Slaughter D. A syndrome of microangiopathic hemolytic anemia, renal impairment and pulmonary edema in chemotherapy-treated patients with adenocarcinoma. Cancer 1986; 58: 1428-1436.

302. Verweij J, van der Burg MEL, Pinedo HM. Mitomycin Cinduced hemolytic uremic syndrome: six case reports and review of the literature on renal, pulmonary and cardiac side effects of the drug. Radiother Oncol 1987; 8: 33-41.

303. Montes A, Powles TJ, O'Brien MER, Ashley SE, Luckit J, Treleaven J. A toxic interaction between mitomycin $\mathrm{C}$ and tamoxifen causing the haemolytic uraemic syndrome. Eur J Cancer 1993; 29: 1854-1857.

304. Torra R, Poch E, Torras A, Bombi JA, Revert L. Pulmonary hemorrhage as a clinical manifestation of hemolytic-uremic syndrome associated with mitomycin $\mathrm{C}$ therapy. Chemotherapy 1993; 39: 453-456.

305. Matsukawa Y, Takeuchi J, Aiso M, et al. Interstitial pneumonitis possibly due to mitoxantrone. Acta Haematol 1993; 90: 155-158.

306. Brandstetter RD, Tamarin FM, Rangraj MS, Ruiz M, Giampietro J. Moxalactam disodium-induced pulmonary hemorrhage. Chest 1984; 86: 644-645.

307. Levy MB, Fink JN, Guzetta PA. Nadolol and hypersensitivity pneumonitis. Ann Intern Med 1986; 105: 806-807.

308. DesMarteau JK, Cassot AL. Acute pulmonary edema resulting from nalbuphine reversal of fentanyl induced respiratory depression. Anesthesiology 1986; 65: 237.

309. Stadnyk A, Grossman RF. Nalbuphine-induced pulmonary edema. Chest 1986; 90: 773-774.

310. Dan M, Aderka D, Topilsky M, Livni E, Levo Y. Hypersensitivity pneumonitis induced by nalidixic acid. Arch Intern Med 1986; 146: 1423-1424.

311. Partridge BL, Ward CF. Pulmonary edema following low-dose naloxone administration. Anesthesiology 1986; 65: 709-710.

312. Prough DS, Roy R, Bumgarner J, Shannon G. Acute pulmonary edema in healthy teenagers following conservative doses of intravenous naloxone. Anesthesiology 1984; 60 485-486.

313. Salberg DJ, Simon MR. Severe asthma induced by naproxen: a case report and review of the literature. Ann Allergy 1980; 45: 372-375.

314. Allen RW, Holt AH, Brown MG. Acute pulmonary sensitivity to nitrofurantoin. Am J Roentgenol 1968; 104: 784-786. 
315. Bäck O, Lundgren R, Wiman LG. Nitrofurantoin-induced pulmonary fibrosis and lupus syndrome. Lancet 1974; i: 930.

316. Bone RC, Wolfe J, Sobonya RE, et al. Desquamative interstitial pneumonia following chronic nitrofurantoin therapy. Chest 1976; 69: 296-297.

317. Lebecque F, Mairesse M. Pneumopathie à la nitrofurantoïne. Poumon Coeur 1983; 39: 101-108.

318. Rosenow ECI, DeRemee RA, Dines DE. Chronic nitrofurantoin pulmonary reaction: report of five cases. $N$ Engl J Med 1968; 279: 1258-1262.

319. Selroos O, Edgren J. Lupus-like syndrome associated with pulmonary reaction to nitrofurantoin. Acta Med Scand 1975; 197: 125-129.

320. Stein JJ, Martin DC. Nitrofurantoin pulmonary hypersensitivity reaction. J Urol 1973; 110: 577-578.

321. Strandberg I, Wengle B, Fagrell B. Chronic interstitial pneumonitis with fibrosis during long-term treatment with nitrofurantoin. Acta Med Scand 1974; 196: 483487.

322. Difederico EM, Harrison M, Matthay MA. Pulmonary edema in a woman following fetal surgery. Chest 1996; 109: $1114-1117$.

323. Lombard CM, Churg A, Winokur S. Pulmonary venoocclusive disease following therapy for malignant neoplasms. Chest 1987; 92: 871-876.

324. Hamm H, Aumiller J, Bohmer R, Missmahl HP, Fullbrandt U, Frenzel H. Alveolitis associated with nomifensine. Lancet 1985; i: 1328-1329.

325. Salmeron S, Brochard L, Rain B, et al. Early neutrophil alveolitis after rechallenge in drug-induced alveolitis. Thorax 1988; 43: 647-648.

326. Beji M, Louzir B, Mahouachi R, El Mekki F, Laribi H, Daghfous J. Oedème aigu du poumon secondaire à la Baralgine. Rev Mal Resp 1995; 12 (Suppl. 2): R97-R98.

327. Williams IP, Millard FJC. Severe asthma after inadvertent ingestion of oxprenolol. Thorax 1980; 35: 160.

328. Broquetas J, Aran X, Soler J. Oxprenolol-induced lifethreatening bronchospasm. Chest 1985; 87: 555-556.

329. Cameron DC. Diffuse pulmonary disorder caused by oxyphenbutazone. $\mathrm{Br}$ Med $J$ 1975; ii: 500-501.

330. Goldberg HL, Vannice SB. Pneumonitis related to treatment with paclitaxel. J Clin Oncol 1995; 13: 534.

331. Ramanathan RK, Belani CP. Transient pulmonary infiltrates: a hypersensitivity reaction to Paclitaxel. Ann Intern Med 1996; 124: 278.

332. Harle TS, Kountoupis JT, Boone MLM, Fred HL. Pulmonary edema without cardiomegaly. Am J Roentgenol 1968; 103: 555-560.

333. Bréchot JM, Buy JN, Laaban JP, Rochemaure J. Computed tomography and magnetic resonance findings in lipoid pneumonia. Thorax 1991; 46: 738-739.

334. Carrillon Y, Tixier E, Revel D, Cordier JF. MR diagnosis of lipoid pneumonia. J Comput Assist Tomogr 1988; 12: 876-877.

335. Dalphin JC, Breton JL, Dubiez A, Ranfaing E, Depierre A, Girardel JM. Deux cas de paraffinose pulmonaire. Rev Med Interne 1988; 9: 410-413.

336. Lee KS, Müller NL, Hale V, Newell JD, Lynch DA, Im JG. Lipoid pneumonia: CT findings. J Comput Assist Tomogr 1995; 19: 48-51.

337. Wheeler PS, Stitik FP, Hutchins GM, Klinefelter HF, Siegelman SS. Diagnosis of lipoid pneumonia by computed tomography. J Am Med Assoc 1981; 245: 65-66.

338. Camus P. Manifestations respiratoires associées aux traitements par la d-pénicillamine. Rev Fr Mal Respir 1982; 10: 7-20.
339. Camus P. A propos des bronchiolites oblitérantes. Rev Fr Mal Respir 1988; 5: 193-194.

340. Falk MS, Newcomer VD. Loeffler's syndrome: occurrence in two patients treated with penicillin in oil and wax. J Am Med Assoc 1949; 141: 21-22.

341. Reichlin S, Loveless MH, Kane EG. Loeffler's syndrome following penicillin therapy. Ann Intern Med 1953; 38: 113-120.

342. James LP, Austen KF. Fatal systemic anaphylaxis in man. N Engl J Med 1964; 270: 597-603.

343. McCombs RP. Systemic "allergic" vasculitis: clinical and pathological relationships. J Am Med Assoc 1965; 194: $157-162$.

344. Molina C, Brun J, Bernard-Griffiths I. Infiltrats pulmonaires à éosinophiles et allergie à la pénicilline. Rev Tuberc Pneumol 1972; 36: 923-932.

345. Wengrower D, Tzfoni EE, Drenger B, Leitersdorf E. Erythroderma and pneumonitis induced by penicillin? Respiration 1986; 50: 301-303.

346. Ducoloné A, Vandevenne A, Ringwald P, Burghard G. Poumon éosinophile d'origine médicamenteuse. Semin Hôp Paris 1986; 62: 1107-1110.

347. Yonemaru M, Mizuguchi Y, Kasuga I, Utsumi K, Ichinose Y, Toyama K. Hilar and mediastinal lymphadenopathy with hypersensitivity pneumonitis induced by penicillin. Chest 1992; 102: 1907-1909.

348. Dupon M, Malou M, Rogues AM, Lacut JY. Acute eosinophilic pneumonia induced by inhaled pentamidine isothionate. Br Med J 1993; i: 109.

349. Gearhart MO, Bhutani MS. Intravenous pentamidineinduced bronchospasm. Chest 1992; 102: 1891-1892.

350. Nevins M, Berque S, Corwin N, Lyon L. Phenylbutazone and pulmonary oedema. Lancet 1969; ii: 1358.

351. Thurston JGB, Marks P, Trapnell D. Lung changes associated with phenylbutazone treatment. Br Med J 1976; 3: 1422-1423.

352. Goldstein G. Sarcoid reaction associated with phenylbutazone hypersensitivity. Ann Intern Med 1963; 59: 97-100.

353. Heitzman ER. Lymphadenopathy related to anticonvulsant therapy: roentgen findings simulating lymphoma. Radiology 1967; 89: 311-312.

354. Bayer AS, Targan SR, Pitchon HE, Guze LB. Dilantin toxicity: miliary pulmonary infiltrates and hypoxemia. Ann Intern Med 1976; 85: 475-476.

355. Michael JR, Rudin ML. Acute pulmonary disease caused by phenytoin. Ann Intern Med 1981; 95: 452-454.

356. Yermakov VM, Hitti IF, Sutton AL. Necrotizing vasculitis associated with diphenylhydantoin: two fatal cases. Hum Pathol 1983; 14: 182-184.

357. Chamberlain DW, Hyland RH, Ross DJ. Diphenylhydantoininduced lymphocytic interstitial pneumonia. Chest 1986; 90: 458-460.

358. Mahatma M, Haponik EF, Nelson S, Lopez A, Summer WR. Phenytoin-induced acute respiratory failure with pulmonary eosinophilia. Am J Med 1989; 87: 93-94.

359. Angabeen SK, Dadparvar S, Brown SJ, et al. The role of gallium-67-citrate in the detection of phenytoin-induced pneumonitis. J Nucl Med 1994; 35: 471-473.

360. Murphy D, Kronick JB, Rieder MJ. Acute respiratory failure mediated by reactive drug metabolites. Biol Neonate 1995; 67: 223-229.

361. Krumhaar D, Urban M, Stempinski E, Otto H. Die sogenannte Hypophysenschnupferlunge. Dtsch Med Wschr 1988; 113: 857-859.

362. Hastier P, Bellon S, Perrin C, et al. Fatal adult respiratory distress syndrome following polyethylene glycol 
administration for bowel cleaning prior to colonoscopy. Gastroenterol Clin Biol 1996; 20: 216-217.

363. Marshall AJ, Barritt DW, Griffiths DA, Laszlo G. Respiratory disease associated with practolol therapy. Lancet 1977; ii: 1254-1257.

364. Azher M, EI-Kassimi FA, Wright SG, Mofti A. Exudative polyserositis and acute respiratory failure following praziquantel therapy. Chest 1990; 98: 241-243.

365. Asherson RA, Zulman J, Hugues GRV. Pulmonary thromboembolism associated with procainamide-induced lupus syndrome and anticardiolipin antibodies. Ann Rheum Dis 1989; 48: 232-235.

366. Goldberg SK, Lipschutz JB, Ricketts RM, Fein AM. Procainamide-induced lupus lung disease characterized by neutrophil alveolitis. Am J Med 1984; 76: 146-150.

367. Klimas NG, Patarca R, Perez G, et al. Case report: distinctive immune abnormalities in a patient with procainamide-induced lupus and serositis. $\mathrm{Am} \mathrm{J} \mathrm{Med} \mathrm{Sci}$ 1992; 303: 99-104

368. Kaplan JM, Wachtel HL, Czarnecki SW, Sampson JJ. Lupus-like illness precipitated by procainamide hydrochloride. J Am Med Assoc 1965; 192: 444-447.

369. Dohner VA, Ward HP, Stanford RE. Alveolitis during procarbazine, vincristine and cyclophosphamide therapy. Chest 1972; 62: 636-638.

370. Farney RJ, Morris AH, Armstrong JD, Hammer S. Diffuse pulmonary disease after therapy with nitrogen mustard, vincristine, procarbazine and prednisone. Am Rev Respir Dis 1977; 115: 135-145.

371. Jones SE, Moore M, Blank N, Castellino RA. Hypersensitivity to procarbazine (Matulane ${ }^{\circledR}$ ) manifested by fever and pleuropulmonary reaction. Cancer 1972; 29: 498-500.

372. Lewis LD. Procarbazine associated alveolitis. Thorax 1984; 39: 206-207.

373. Lokich JJ, Moloney WC. Allergic reaction to procarbazine. Clin Pharmacol Ther 1972; 13: 573-574.

374. Olm M, Munne P, Jimenez MJ. Severe reactive airways disease induced by propafenone. Chest 1989; 95: 1366-1367.

375. Bogartz LJ, Miller WC. Pulmonary edema associated with proproxyphen intoxication. J Am Med Assoc 1971; 215: 259.

376. Ahmad S. Sclerosing peritonitis and propranolol. Chest 1981; 79: 361-362.

377. Akoun G, Milleron BJ, Mayaud CM, Tholonat D. Provocation test coupled with bronchoalveolar lavage in diagnosis of propranolol-induced hypersensitivity pneumonitis. Am Rev Respir Dis 1989; 139: 247-249.

378. Cassorla FG, Finegold DN, Parks JS, Tenore A, Thawenari H, Baker L. Vasculitis; pulmonary cavitation, and anemia during antithyroid drug therapy. Am J Dis Child 1983; 137: 118-122.

379. Chevrolet JC, Guelpa G, Schifferli JA. Recurrent adult respiratory distress-like syndrome associated with propylthiouracil therapy. Eur Respir J 1991; 4: 899-901.

380. Middleton KL, Santella R, Couser JI Jr. Eosinophilic pleuritis due to propylthiouracil. Chest 1993; 103: 955-956.

381. Stankus SJ, Johnson NT. Propylthiouracil-induced hypersensitivity vasculitis presenting as respiratory failure. Chest 1992; 102: 1595-1596.

382. Fishburne JI Jr, Brenner WE, Braaksma JT, Hendricks $\mathrm{CH}$. Bronchospasm complicating intravenous prostaglandin F2 $\alpha$ for therapeutic abortion. Obstet Gynecol 1972; 39: 892-896.

383. Morel DR, Lowenstein E, Nguyenduy T, et al. Acute pulmonary vasoconstriction and thromboxane release during protamine reversal of heparin anticoagulation in awake sheep. Circ Res 1988; 62: 905-915.
384. Murphy RL, Phair JP. Systemic reaction to pyrimethamine-sulfadoxine. J Fam Pract 1986; 22: 375-376.

385. McCormack D, Morgan WKC. Fansidar hypersensitivity pneumonitis. Br J Dis Chest 1987; 81: 194-196.

386. Davidson AC, Bateman C, Shovlin C, Marrinan M, Burton GH, Cameron IR. Pulmonary toxicity of malaria prophylaxis. $B r$ Med J 1988; ii: 1240-1241.

387. Pang JA. Noncardiogenic pulmonary oedema associated with pyrimethamine. Respir Med 1989; 83: 247-248.

388. Poukkula A, Päkkö P. Quinidine-induced reversible pneumonitis. Chest 1994; 106: 304-306.

389. Alperin JB, de Groot WJ, Cimo PL. Quinidine-induced thrombocytopenia with pulmonary hemorrhage. Arch Intern Med 1980; 140: 266-267.

390. Kramer MR, Estenne M, Berkman N, et al. Radiationinduced pulmonary veno-occlusive disease. Chest 1993; 104: 1282-1284.

391. Ma LD, Taylor GA, Wharam MD, Wiley JM. Recall pneumonitis: adriamycin potentiation of radiation pneumonitis in two children. Radiology 1993; 187: 465-467.

392. Perrin C, Mouroux J, Tamisier R, Vandenbos F, Dumon MC, Blaive B. Un chylothorax d'origine particulière: la fibrose médiastino-pulmonaire post-radique. Presse Méd 1996; 25: 259.

393. Davis SD, Yankelevitz DF, Henschke CI. Radiation effects on the lung: clinical features, pathology, and imaging findings. Am J Roentgenol 1992; 159: 1157-1164.

394. Polliack A. Late therapy-induced cardiac and pulmonary complications in cured patients with Hodgkin's disease treated with conventional combination chemoradiotherapy. Leuk Lymph 1995; 15 (Suppl. 1): 7-10.

395. Morrone N, Gama e Silva Volpe VL, Dourado AM, Mitre F, Coletta ENAM. Bilateral pleural effusion due to mediastinal fibrosis induced by radiotherapy. Chest 1993; 104: 1276-1278.

396. Rowinsky EK, Abeloff MD, Wharam MD. Spontaneous pneumothorax following thoracic radiation. Chest 1985; 88: 703-708.

397. Byhardt RW, Abrams R, Almagro U. The association of adult respiratory distress syndrome (ARDS) with thoracic irradiation (RT). Int J Radiat Oncol Biol Phys 1988; 50: 1441-1446.

398. Frankel SR, Eardley A, Lauwers G, Weiss M, Warrell RP Jr. The "retinoic acid syndrome" in acute promyelocytic leukemia. Ann Intern Med 1992; 117: 292-296.

399. Smith-Whitley K, Lange B. Fatal all-trans retinoic acid pneumonitis. Ann Intern Med 1993; 118: 472-473.

400. Wiley JS, Firkin FC. Reduction of pulmonary toxicity by prednisolone prophylaxis during all-trans retinoic acid treatment of acute promyelocytic leukemia. Leukemia 1995; 9: 774-778.

401. Degroot REB, Willems LNA, Dijkman JH. Interstitial lung disease with pleural effusion caused by simvastin. J Intern Med 1996; 239: 361-363.

402. Bodman SF, Condemi JJ. Mediastinal widening in iatrogenic Cushing's syndrome. Ann Intern Med 1967; 67: 399-403.

403. Bowyer SL, LaMothe MP, Hollister JR. Steroid myopathy: incidence and detection in a population with asthma. J Allergy Clin Immunol 1985; 76: 234-242.

404. Kernstein MD, Adinolfi MF. Pulmonary dysfunction associated with streptokinase therapy. Arch Surg 1986; 121: 852-853.

405. Cuthbert RT. Löffler's syndrome occurring during streptomycin and PAS therapy. Br Med J 1954; ii: 398-399.

406. Klinghoffer J. Löffler's syndrome following use of vaginal cream. Ann Intern Med 1954; 40: 343-350. 
407. Koch-Weser J, Sidel VW, Dexter M, Parish C, Finer DC, Kanarek P. Adverse reactions to sulfisoxazole, sulfamethoxazole, and nitrofurantoin: manifestations and specific reaction rates during 2,118 courses of therapy. Arch Intern Med 1971; 128: 399-404.

408. Shear NH, Spielberg SP, Grant DM, Tang BK, Kalow W. Differences in metabolism of sulfonamides predisposing to idiosyncratic toxicity. Ann Intern Med 1986; 105: 179-184.

409. Moss SF, Ind PW. Time course of recovery of lung function in sulfasalazine-induced alveolitis. Respir Med 1991; 85: 73-75.

410. Wang KK, Bowyer BA, Fleming CR, Schroeder KW. Pulmonary infiltrates and eosinophilia associated with sulfasalazine. Mayo Clin Proc 1984; 59: 343-346.

411. Yamakado S, Yoshida Y, Yamada T, Kishida T, Kobayashi M, Nomura T. Pulmonary infiltration and eosinophilia associated with sulfasalazine therapy for ulcerative colitis: a case report and review of the literature. Intern Med 1992; 31: 108-113.

412. Farre JM, Perez T, Hautefeuille P, Tonnel F, Duquesnoy $\mathrm{B}$, Delcambre B. Pleurésie à éosinophiles induite par la salazosulfapyridine. Presse Méd 1989; 18: 987-988.

413. Salemo SM, Ormseth EJ, Roth BJ, Meyer CA, Christensen ED, Dillard TA. Sulfasalazine pulmonary toxicity in ulcerative colitis mimicking clinical features of Wegener's granulomatosis. Chest 1996; 110: 556-559.

414. Smith FE, Lindberg PJ. Life-threatening hypersensitivity to sulindac. J Am Med Assoc 1980; 244: 269270.

415. Fein M. Sulindac and pneumonitis. Ann Intern Med 1981; 95: 245.

416. Park GD, Spector R, Headstream T, Goldberg M. Serious adverse reactions associated with sulindac. Arch Intern Med 1982; 142: 1292-1294.

417. Takimoto CH, Lynch D, Stulbarg MS. Pulmonary infiltrates associated with sulindac therapy. Chest 1990; 97 : 230-232.

418. Smith RP, Dewar JA, Winter JH. Tamoxifen-induced asthma. Lancet 1993; i: 772.

419. Domz CA, McNamara DH, Holzapfel HF. Tetracycline provocation in lupus erythematosus. Ann Intern Med 1959; 50: 1217-1226.

420. Ho D, Tashkin DP, Bein ME, Sharma O. Pulmonary infiltrates with eosinophilia associated with tetracycline. Chest 1979; 76: 33-36.

421. Chiffoleau A, Delavaud P, Cellerin L, Chailleux E, Larousse C. Tiopronine (Acadione) et pneumopathie interstitielle. Therapie 1993; 48: 486-487.

422. Demaziere A, Maugars Y, Chollet S, Prost A. Nonfatal bronchiolitis obliterans possibly associated with tiopronin: a case report with long term follow-up. $\mathrm{Br} J$ Rheumatol 1993; 32: 172-174.

423. Kuei JH, Tashkin DP, Figlin RA. Pulmonary toxicity of recombinant human tumor necrosis factor. Chest 1989; 96: 334-338.

424. Schilling PJ, Murray JL, Markowitz AB. Novel tumor necrosis factor toxic effects: pulmonary hemorrhage and severe hepatic dysfunction. Cancer 1992; 69: 256-260.

425. Stromberg C, Palva E, Alhava E, Aranko K, IdanpaanHeikkila J. Pulmonary infiltrations induced by tolfenamic acid. Lancet 1987; ii: 685.

426. Strauss RG, Connett JE, Gale RP, et al. A controlled trial of prophylactic granulocyte transfusions during initial induction chemotherapy for acute myelogenous leukemia. N Engl J Med 1981; 305: 597-603.

427. Chan TH, Ho SS, Li PKT. Noncardiogenic pulmonary edema associated with triazolam. Clin Toxicol 1995; 33 : $185-187$.

428. Hatzinger M, Stohler R, Hoslboer-Trachsler E. Eosinophile pleuritis und Hepatopathie unter Trimipramin-Therapie. Schweiz Med Wschr 1991; 121: 910-912.

429. Bogaerts Y, van Rentenghem D, Vanvuchelen J, et al. Interstitial pneumonitis and pulmonary vasculitis in a patient taking an 1-tryptophan preparation. Eur Respir J 1991; 4: 1033-1036.

430. Campagna AC, Blanc PD, Criswell LA, et al. Pulmonary manifestations of the eosinophilia-myalgia syndrome associated with tryptophan ingestion. Chest 1992; 101: 1274-1281.

431. Strumpf IJ, Drucker RD, Anders KH, Cohen S, Fajolu O. Acute eosinophilic pulmonary disease associated with ingestion of 1-tryptophan-containing products. Chest 1991; 99: 8-13.

432. Yakovlevitch M, Siegel M, Hoch DH, Rutlen DL. Pulmonary hypertension in a patient with tryptophaninduced eosinophilia myalgia. Am J Med 1991; 90: 272-273.

433. Matsis P, Mann S. Rigors and bronchospasm with urokinase after streptokinase. Lancet 1992; ii: 1552.

434. Tulandi T, Beique F, Kimia M. Pulmonary edema: a complication of local injection of vasopressin at laparoscopy. Fertil Steril 1996; 66: 478-480.

435. Figueredo AT, Jones G, Kay MJ, Higgins D, Young JEM. Kaposi's sarcoma of the lung; remission followed by fatal pneumonitis after vinblastine and thoracic irradiation. Acta Oncol 1995; 34: 532-533.

436. Rao SX, Ramaswany G, Levin M, McCravey JW. Fatal acute respiratory failure after vinblastine-mitomycine therapy in lung carcinoma. Arch Intern Med 1985; 145 : 1905-1907.

437. Franchi F, Seminara P, Gualdi GF. Lung pseudometastases due to vinblastine toxicity. Klin Wschr 1984; 62: 1047-1048.

438. Kris MG, Pablo D, Gralla RJ, Burke MT, Prestifilippo $\mathrm{J}$, Lewin D. Dyspnea following vinblastine or vindesine administration in patients receiving mitomycin plus vinca alkaloid combination therapy. Cancer Treat Rep 1984; 68: 1029-1031.

439. Isawa T, Ono R, Motomiya M, Tamahashi N. Fatal acute interstitial pneumonia induced by low-dose doxorubicin and vindesine. Tohoku J Exp Med 1989; 158: 149-154.

440. Vaylet F, Plotton C, Algayres JP, Verkindre C, L'Her $\mathrm{P}$. Oedème aigu du poumon après vinorelbine. Presse Méd 1996; 25: 1259-1260.

441. Bauer JM, Freyberg RH. Vitamin D intoxication with metastatic calcification. J Am Med Assoc 1946; 130: 1208-1215.

442. Barnett VT, Bergmann F, Humphrey H, Chediak J. Diffuse alveolar hemorrhage secondary to superwarfarin ingestion. Chest 1992; 102: 1301-1302.

443. Moncada RM, Venta LA, Venta ER, Fareed J, Walenga JM, Messmore HL. Tracheal and bronchial cartilaginous rings: warfarin sodium-induced calcification. Radiology 1992; 184: 437-439.

444. Cohen AF, Warman SP. Upper airway obstruction secondary to warfarin-induced sublingual hematoma. Arch Otolaryngol 1989; 115: 718-720.

445. Reussi C, Schiavi JE, Altman R, Yussem EE, Rouvier J. Unusual complications in the course of anticoagulant therapy. Am J Med 1969; 46: 460-463.

446. Pleskow WW, Lumry WR, Simon RA. Zomepirac sodium (Zomax) hypersensitivity in aspirin-sensitive asthmatics. Am Rev Respir Dis 1984; 130: 1170-1173. 